JOURNAL OF THE

AMERICAN MATHEMATICAL SOCIETY

Volume 17, Number 2, Pages 267-296

S 0894-0347(04)00449-7

Article electronically published on January 7, 2004

\title{
FOLIATIONS IN MODULI SPACES OF ABELIAN VARIETIES
}

\author{
FRANS OORT
}

\section{INTRODUCTION}

In this paper we study abelian varieties and $p$-divisible groups in characteristic $p$.

Even though a non-trivial deformation of an abelian variety can produce a nontrivial Galois-representation, say on the Tate- $\ell$-group of the generic fiber (in any characteristic), the geometric generic fiber has a "constant" Tate- $\ell$-group. We encounter the same phenomenon for the $p$-structure in positive characteristic: any two ordinary abelian varieties of the same dimension over an algebraically closed field have isomorphic $p$-divisible groups.

However, for non-ordinary abelian varieties this seems to break down. The fascinating structure which comes out of this is that the maximal locus where a given geometric isomorphism class of a $p$-divisible group is realized (e.g., in a family of abelian varieties) is a locally closed set. The locus defined by the geometric isomorphism type of a $p$-divisible group will be called a "central leaf"; see 2.1 and 3.4 This gives rise to a "foliation" of the open stratum attached to a Newton polygon $\xi$; the dimension of any "leaf" in the same Newton polygon stratum solely depends on $\xi$. In extreme cases

either the leaf is the whole stratum, as in the ordinary case, or in the "almost ordinary case" (the $p$-rank equals $g-1$ ),

or a leaf is zero-dimensional as in the supersingular case;

in intermediate cases a leaf can be a proper subset and still be positive dimensional: we have worked out the example of $g=4$ in 8.1

Between two central leaves in the same Newton polygon stratum there is a correspondence by iterated $\alpha_{p}$-isogenies. This leads naturally to the study of "Hecke orbits" by such isogenies. In the case of moduli spaces of abelian varieties we obtain "isogeny leaves"; see 4.2 The beauty of these two structures is that they are almost transversal, that both feel like a foliation, and that they give, up to a finite morphism, a natural product structure on every irreducible component $W$ of an open Newton polygon stratum.

We prove the following theorems:

1.3: a finite level of a geometrically fiberwise constant family of $p$-divisible groups becomes constant over an appropriate finite cover of the base;

2.2, 3.3 a central leaf is closed in its open Newton polygon stratum;

Received by the editors June 16, 2002.

2000 Mathematics Subject Classification. Primary 14K10, 14L05. 
3.13 central leaves are smooth over the base field, and the dimension of a central leaf depends only on the Newton polygon;

4.2. for polarized abelian varieties, the union of all irreducible iterated $\alpha_{p^{-}}$ Hecke orbits through one point is a closed subset;

5.3. every component of a Newton polygon stratum is up to a finite morphism isomorphic with the product of any of the isogeny leaves with a finite cover of any of the central leaves.

Motivation/explanation. In the moduli space of abelian varieties in characteristic $p$ we consider Hecke orbits related to isogenies of degree prime to $p$ and Hecke orbits related to iterated $\alpha_{p}$-isogenies. The first "moves" points in a central leaf: under such isogenies the geometric $p$-divisible group does not change; the second moves points in an isogeny leaf. We can expect that these two natural foliations describe these two "transversal" actions: see 6.1 and 6.2

EO-strata and central leaves. In [18] we described the stratification of the moduli space of abelian varieties given by $p$-kernels: $(A, \lambda)$ and $(B, \mu)$ are in the same EO-stratum if $(A, \lambda)[p] \cong(B, \mu)[p]$, the isomorphism over an algebraically closed field. It is natural to consider more generally the relation given by the isomorphism type of $(A, \lambda)\left[p^{i}\right]$ for some $i \in \mathbb{Z}_{\geq 1}$; for every $i$ this defines naturally subsets of the moduli space, but we should not expect only finitely many of such subspaces. The central leaves studied in this paper are the ones obtained by choosing $i$ large enough (depending on $g$ ), which is the same (see 1.7) as considering the isomorphism type of $(A, \lambda)\left[p^{\infty}\right]$.

Isogeny correspondences. Such correspondences are finite-to-finite in characteristic zero or, more generally, if only isogenies of degree prime to the characteristic are considered. In positive characteristic such correspondences in general blow up and down. However it turns out that restricted to central leaves all correspondences are finite-to-finite; see 3.16 .

Some terminology and notations used in this paper are brought together in Section [7 and Section 8. In particular a field denoted by $k$ will be supposed algebraically closed, $k=\bar{k} \supset \mathbb{F}_{p}$.

\section{Preliminaries on $p$-Divisible groups and on finite group SChemes}

1.1. Definition. Let $S$ be a scheme, and let $X \rightarrow S$ be a $p$-divisible group. We say that $X / S$ is geometrically fiberwise constant if there exist a field $K$, a $p$-divisible group $X_{0}$ over $K$, a morphism $S \rightarrow \operatorname{Spec}(K)$, and for every $s \in S$ an algebraically closed field $k \supset \kappa(s) \supset K$ containing the residue class field of $s$ and an isomorphism $X_{0} \otimes k \cong_{k} X_{s} \otimes k$.

The analogous terminology will be used for (polarized) abelian schemes.

1.2. Definition. Let $T$ be a scheme and let $G \rightarrow T$ be a group scheme. We say that $G$ is constant over $T$ if there exist a field $L$, a group scheme $G_{0}$ over $L$, a morphism $T \rightarrow \operatorname{Spec}(L)$ and an isomorphism

$$
G_{0} \times_{\operatorname{Spec}(L)} T \cong \cong_{T} \quad G .
$$

In this section we show 
1.3. Theorem. Let $S$ be a scheme which satisfies condition (N) (see 7.2); let $\mathcal{X} \rightarrow S$ be a p-divisible group; let $n \in \mathbb{Z}_{\geq 0}$. Suppose that $\mathcal{X} \rightarrow S$ is geometrically fiberwise constant. Then there exists a finite surjective morphism $T_{n}=T \rightarrow S$, such that $\mathcal{X}\left[p^{n}\right] \times{ }_{S} T$ is constant over $T$.

Remark. The choice of $T \rightarrow S$ can be made in such a way that this equals a composition of finite morphisms $T \rightarrow T^{\prime} \rightarrow S^{\prime} \rightarrow S$, where $S^{\prime} \rightarrow S$ factors the normalization, $\tilde{S} \rightarrow S^{\prime} \rightarrow S$, and $T^{\prime} \rightarrow S^{\prime}$ is purely inseparable and $T \rightarrow T^{\prime}$ is étale.

For the proof of 1.3 we need some preliminaries. We say that the condition $*(Z / S, n)$ is satisfied, where $Z \rightarrow S$ is a p-divisible group, and $n \in \mathbb{Z}_{\geq 0}$, if there exists a finite surjective morphism $T \rightarrow S$ such that $Z\left[p^{n}\right] \times_{S} T$ is constant.

1.4. Lemma. For any completely slope divisible $Y \rightarrow S$, where $S$ is noetherian and integral, the property $*(Z / S, N)$ is satisfied for every $N \in \mathbb{Z}_{\geq 0}$.

Proof. At first we claim that there exist a purely inseparable, finite, surjective morphism $T_{1}=T \rightarrow S$ such that $Y\left[p^{N}\right]_{T}=\oplus_{i}\left(\left(Y_{i} / Y_{i-1}\right)\left[p^{N}\right]\right)_{T}$, in the notation of 7.6.

In fact, for large $r \in \mathbb{Z}$, over $T^{\prime}=S^{\left(p^{-r s}\right)}$ the homomorphism

$$
\left(\psi:=\frac{F^{s}}{p^{s-t_{i}}}\right)^{r}:\left(\left(Y_{i} / Y_{i-1}\right)\left[p^{N}\right]\right)_{T^{\prime}}^{\left(p^{-r s}\right)} \longrightarrow Y_{i}\left[p^{N}\right]_{T^{\prime}}
$$

splits off this subquotient, because $\psi$ is an isomorphism on $Y_{i} / Y_{i-1}$ and nilpotent on $Y_{i-1}\left[p^{N}\right]$. Note that $S$ is integral and noetherian, and $Y_{i}\left[p^{N}\right] \rightarrow S$ is finite; hence there exists a factorization $T^{\prime}=S^{\left(p^{-r s}\right)} \rightarrow T_{1} \rightarrow S$, where $T_{1} \rightarrow S$ is finite and for which the claim holds.

By [22, 1.1.0] we see that all $G_{i}=\left(Y_{i} / Y_{i-1)}\left[p^{N}\right]\right.$ become constant, for $1 \leq i \leq m$, under a (separable) finite, surjective morphism $T_{2} \rightarrow S$. Any $T \rightarrow S$ dominating $T_{1} \rightarrow S$ and $T_{2} \rightarrow S$ has the desired property. This proves the lemma.

For $p$-divisible groups $X, Y$ over a field and for $N \geq n \geq 0$ we denote by

$$
\Phi_{n}: \operatorname{Hom}(X, Y) \rightarrow \operatorname{Hom}(X[n], Y[n])
$$

and

$$
\Phi_{n}^{N}: \operatorname{Hom}(X[N], Y[N]) \rightarrow \operatorname{Hom}(X[n], Y[n])
$$

the natural restriction maps.

1.5. Lemma. For $p$-divisible groups $X$ and $Y$ over $k$, there exists for every $n \in \mathbb{Z}_{\geq 0}$ an integer $N(X, Y, n)$ such that for every $N \geq N(X, Y, n)$ we have

$$
\operatorname{Im}\left(\Phi_{n}\right)=\operatorname{Im}\left(\Phi_{n}^{N}\right) .
$$

Proof. For every $m \in \mathbb{Z}_{\geq 0}$ consider the functor $T \mapsto \operatorname{Hom}\left(X\left[p^{m}\right]_{T}, Y\left[p^{m}\right]_{T}\right)$ on $k$-schemes. As is easily seen, this functor is representable by a group scheme of finite type over $k$; notation: $G_{m}=\underline{\operatorname{Hom}}\left(X\left[p^{m}\right], Y\left[p^{m}\right]\right)$. For $m \geq n$ the natural restriction map yields a homomorphism of algebraic groups $\rho_{n}^{m}: G_{m} \rightarrow G_{n}$. We write $G_{m}^{\prime}=\left(G_{m}\right)_{\text {red }}$. Note that the image of a homomorphism between algebraic groups is a (closed) subgroup; consider the finite set $\Phi_{n}(\operatorname{Hom}(X, Y)) \subset G_{n}^{\prime}(k)$ as an algebraic subgroup of $G_{n}^{\prime}$. We obtain a descending chain of algebraic groups

$$
G_{n}^{\prime} \supset \rho_{n}^{n+1}\left(G_{n+1}^{\prime}\right) \supset \cdots \supset \rho_{n}^{n+i}\left(G_{n+i}^{\prime}\right) \supset \cdots \supset \Phi_{n}(\operatorname{Hom}(X, Y)) .
$$


The sequence of subgroup schemes $\left\{\rho_{n}^{n+i}\left(G_{n+i}^{\prime}\right) \mid i \in \mathbb{Z}_{\geq 0}\right\}$ stabilizes on the one hand, and it is equal to $\Phi_{n}(\operatorname{Hom}(X, Y))$ on the other hand.

We are going to show that $n \mapsto N(X, Y, n)$ can be chosen uniformly depending only on the heights of the $p$-divisible groups in consideration.

1.6. Proposition. For every $h \in \mathbb{Z}_{>0}$ there exists an integer $N\left(h^{\prime}, h^{\prime \prime}, n\right)$ such that for $p$-divisible groups $X$ and $Y$ over an algebraically closed field $k$ of height $h^{\prime}=\operatorname{height}(X)$, respectively $h^{\prime \prime}=$ height $(Y)$, and for every $N \geq N\left(h^{\prime}, h^{\prime \prime}, n\right)$ the following images are equal:

$$
\operatorname{Im}\left(\Phi_{n}\right)=\operatorname{Im}\left(\Phi_{n}^{N}\right)
$$

Proof. We split up the proof into various steps.

(1) Given $h$, there exists an integer $d(h)$ such that for every $p$-divisible group $X$ of height $h$ and with Newton polygon $\mathcal{N}(X)=\beta$ there is an isogeny $\rho: H(\beta) \rightarrow X$ of degree $\operatorname{deg}(\rho)=d(h)$.

This follows from [12, (3.4) and (3.5) on page 44] and from the property that $H(\beta)$ admits endomorphisms of degree $p$.

(2) In order to prove the proposition it suffices to show:

For every $h \geq 0$ there exists $N(h)$ such that for every $p$-divisible group $Z$ of height $h$ and for every $N \geq N(h)$ we have

$$
\operatorname{Im}\left(\Phi_{n}: \operatorname{End}(Z) \rightarrow \operatorname{End}(Z[n])\right)=\operatorname{Im}\left(\Phi_{n}^{N}: \operatorname{End}(Z(N)) \rightarrow \operatorname{End}(Z[n])\right) .
$$

Indeed, apply this result for $Z=X \oplus Y$.

(3) Let $H$ and $Z$ be $p$-divisible groups and let $\rho: H \rightarrow Z$ be an isogeny of degree $\operatorname{deg}(\rho)=p^{s}$. Suppose that the property mentioned in (2) holds for $H$ with the function $b \mapsto N_{H}(b)$; then that property holds for $Z$ with the function $n \mapsto N_{Z}(n):=$ $N_{H}(n+s)+s$.

Proof of (3). We consider Dieudonné modules and induced maps:

$$
\mathbb{D}(\rho: H \rightarrow Z)=(\mathbb{D}(H)=: Q \hookrightarrow M:=\mathbb{D}(Z)) .
$$

For $n \geq 0$ and $N \geq N_{H}(n+s)+s$ we consider the inclusions:

$$
p^{N} M \subset p^{N_{H}(n+s)} Q \subset p^{n+s} Q \subset p^{n+s} M \subset p^{n} Q \subset Q \subset M \subset p^{-s} Q .
$$

Suppose $\varphi_{N} \in \operatorname{End}(Z[N])$ restricts to $\varphi_{n}=\Phi_{n}^{N}\left(\varphi_{N}\right)$; we claim that in this case $\varphi_{n}$ can be lifted to $\varphi \in \operatorname{End}(Z)$. Indeed, consider $\psi_{n+s} \in \operatorname{End}\left(Z\left[p^{n+s}\right]\right.$ defined by $\psi_{n+s}=p^{s} \varphi_{n}$. We denote the endomorphisms induced on the Dieudonné modules by the same symbols; note that $\psi_{n+s}: M / p^{n+s} M \rightarrow M / p^{n+s} M$ restricts to $\psi_{n+s}^{\prime}$ : $Q / p^{n+s} M \rightarrow Q / p^{n+s} M$ by

$$
\frac{Q}{p^{n+s} M} \hookrightarrow \frac{M}{p^{n+s} M} \stackrel{p^{s} \varphi}{\longrightarrow} \frac{p^{s} M}{p^{n+s} M} \hookrightarrow \frac{Q}{p^{n+s} M} .
$$

We see that $\varphi_{N}$ restricts to $\psi_{N_{H}(n+s)}^{\prime} \in \operatorname{End}\left(H\left[p^{N_{H}(n+s)}\right]\right)=\operatorname{End}\left(Q / p^{N_{H}(n+s)} Q\right)$; hence the restriction to $\operatorname{End}\left(H\left[p^{n+s}\right]\right)$ can be lifted to $H$. This shows that $\psi_{n+s}^{\prime} \in$ $\operatorname{End}\left(Q / p^{n+s} M\right)$ can be lifted to $Q$. From this it follows that $\varphi_{n} \in \operatorname{End}(M)$ can be lifted to $\operatorname{End}(M)$. 
(4) For a given $H=H(\beta)$ there is a function $N_{H}$ such that (2) is satisfied for $H$ and this function. Hence, for all minimal groups of height $h$ there is a function $n \mapsto N_{\min }(h, n)$ satisfying (2) for all minimal groups of height $h$.

The first statement is a consequence of 1.5 . The second follows because there are only finitely many Newton polygons of a given height.

Remark (not used in this paper). An easy and explicit consideration shows that for any Newton polygon $\beta$ the function $N_{H(\beta)}(n):=n+1$ satisfies the property in (4).

We have seen in (2) that it suffices to show the proposition for endomorphisms. For a given $h \in \mathbb{Z}_{>0}$ we define $N(h, n)=N_{\min }(h, n+s)+s$, with $s:=d(h)$ as in (1). By (1) we see that for every $Z$ of height $h$ there exists an isogeny $H=H(\mathcal{N}(Z)) \rightarrow Z$ of degree $d(h)$. By (4) we know that property (2) holds for the function $N_{H}(m)=N_{\min }(h, m)$. By (3) we see that property (2) holds for $Z$ with the function $N_{Z}(n)=N_{\min }(h, n+s)+s$.

Note that the detour via minimal groups is not necessary: the proposition follows, using special Dieudonné submodules as in [12], with a less explicit bound, from [12] (3.4) and (3.5) on page 44], 1.5 and (2) and (3). Note however that the number of completely slope divisible $p$-divisible groups, up to isomorphism over $k$, of given height, in general is not finite; for that reason we did not use these $p$-divisible groups in the previous proof.

The following result will be used several times in this paper. This fact is probably known to all experts in the field.

1.7. Corollary. For any $h \in \mathbb{Z}_{\geq 0}$ there exists $N(h) \in \mathbb{Z}_{\geq 0}$ with the property that for $p$-divisible groups $X_{1}$, and $X_{2}$ of height $h$ over an algebraically closed field, and any $n \geq N(h)$,

Apply [1.6 with $n=1$.

$$
X_{1}\left[p^{n}\right] \cong X_{2}\left[p^{n}\right] \Rightarrow X_{1} \cong X_{2} .
$$

1.8. Corollary. Any completely slope divisible $Y \rightarrow S$ over a noetherian and integral $K$-scheme $S$ with $S(K) \neq \emptyset$ is geometrically fiberwise constant.

This follows from 1.4 and 1.7

1.9. Lemma. Suppose given $X \rightarrow S$, a p-divisible group, and $i \in \mathbb{Z}_{\geq 0}$, then the functor

$$
T \mapsto G r(T):=\left\{G \hookrightarrow X_{T} \mid \operatorname{rk}(G / T)=p^{i}\right\}
$$

is representable by a proper scheme $G r_{X / S, i}=G r \rightarrow S$ and a universal $\mathcal{G} \rightarrow G r$.

Proof. There is a locally free sheaf $\mathcal{B}$ of algebras on $S$ giving $X\left[p^{i}\right] \rightarrow S$. Any $G \hookrightarrow X_{T}$ as considered is included in $X\left[p^{i}\right]_{T}$, and it is given by an ideal sheaf $\mathcal{I}$ in $\mathcal{B} \otimes \mathcal{O}_{T}$. Hence the functor is a subfunctor of the related Grassmannian of $\mathcal{B} / S$. The property that $\mathcal{B} \rightarrow \mathcal{B} / I$ with $G=\operatorname{Spec}(\mathcal{B} / \mathcal{I})$ is in fact a morphism of bialgebras is a closed condition on points in that Grassmannian.

1.10. Lemma. Let $Z_{1}$ and $Z_{2}$ be p-divisible groups over an algebraically closed field $k$, and let $i \in \mathbb{Z}_{\geq 0}$. We write

$$
\Psi=\Psi\left(Z_{1}, Z_{2} ; i\right):=\left\{G \subset Z_{1} \mid \operatorname{rk}(G)=p^{i}, \quad \exists \psi: Z_{1} \rightarrow Z_{2}, \quad \operatorname{Ker}(\psi)=G\right\} ;
$$


then

$$
\#(\Psi)<\infty .
$$

Proof. The module $\Gamma:=\operatorname{Hom}\left(Z_{1}, Z_{2}\right)$ is free of finite rank over $\mathbb{Z}_{p}$. Hence $\Gamma / p^{i} \Gamma$ is finite. Let $\Phi^{\prime} \subset \Gamma$ and let its image $\Phi^{\prime \prime} \subset \Gamma / p^{i} \Gamma$ be defined as

$$
\Phi^{\prime}:=\left\{\psi \in \Gamma \mid \operatorname{rk}(\operatorname{Ker}(\psi))=p^{i}\right\} ; \quad \Phi^{\prime} \bmod p^{i} \Gamma=: \Phi^{\prime \prime} \subset \Gamma / p^{i} \Gamma .
$$

If $\psi_{1} \in \Phi^{\prime}$ and $\rho \in \Phi^{\prime}$ and $\psi_{1}+p^{i} \cdot \rho=: \psi_{2} \in \Phi^{\prime}$, then $\operatorname{Ker}\left(\psi_{1}\right)=\operatorname{Ker}\left(\psi_{2}\right)$. The map $\Phi^{\prime} \rightarrow \Phi$ defined by $\psi \mapsto \operatorname{Ker}(\psi)$ induces a surjective map $\Phi^{\prime \prime} \rightarrow \Psi$; hence $\#(\Psi)<\infty$.

1.11. Proof of Theorem [1.3. Let $S^{\prime} \rightarrow S$ be the normalization morphism, and consider $\mathcal{X}^{\prime}=\mathcal{X}_{S^{\prime}} \rightarrow S^{\prime}$; by [22], Theorem (2.1)] or [22, Proposition (2.7)], we know there exists an isogeny $\varphi: \mathcal{Y} \rightarrow \mathcal{X}^{\prime}$ over $S^{\prime}$, where $\mathcal{Y} / S^{\prime}$ is completely slope divisible. Let $p^{i}:=\operatorname{deg}(\varphi)$. We are going to show $*\left(\mathcal{X}_{S^{\prime}}, n\right)$. We choose $N \in \mathbb{Z}_{>0}$ such that $N \geq n+i$. As $\mathcal{Y} \rightarrow S^{\prime}$ satisfies $*(\mathcal{Y}, N)$ (see 1.4), we can choose a finite $T \rightarrow S^{\prime}$, with $T$ connected, a field $L$, a morphism $T \rightarrow \operatorname{Spec}(L)$, a $p$-divisible group $Y_{0}$ over $L$, and an isomorphism

$$
Y_{0}\left[p^{N}\right] \times T \stackrel{\sim}{\longrightarrow} \mathcal{Y}\left[p^{N}\right] \times_{S^{\prime}} T ;
$$

from now on we fix this isomorphism and write it as an equality. We write $X_{0}=$ $\left(\mathcal{X}_{T}\right)_{0}$, and we have the morphism $\varphi_{T, 0}: Y_{0} \rightarrow X_{0}$. Because $N \geq i$ we see that $\operatorname{Ker}(\varphi) \subset \mathcal{Y}\left[p^{i}\right] \subset \mathcal{Y}\left[p^{N}\right]$; hence $G r:=G r \mathcal{Y}_{, i}=G r_{Y_{0}, i} \times T$. Consider the finite set $\Psi=\Psi\left(Z_{1}=Y_{0}, Z_{2}=X_{0} ; i\right)$ as in 1.10 .

The morphism $\varphi_{T}: \mathcal{Y}_{T} \rightarrow \mathcal{X}_{T}$ defines $\mathcal{G}:=\operatorname{Ker}\left(\varphi_{T}\right) \subset \mathcal{Y}\left[p^{N}\right]_{T}=Y_{0} \times T$; this is equivalent to a section

$$
\text { Gr } \stackrel{\stackrel{\sigma}{\leftrightarrows}}{\longrightarrow} T .
$$

Note that $\mathcal{Y}_{T} / T$ is completely slope divisible; hence for every geometric point $t$ of $T$ there exists some isomorphism $\mathcal{Y}_{t} \cong Y_{0} \otimes k$ (see 1.8); also we have some isomorphism $\mathcal{X}_{t} \cong X_{0} \otimes k$. Hence for every geometric point $t$ of $T$ we have $\sigma(t) \in$ $\Psi=\Psi\left(Y_{0}, X_{0} ; i\right)$; note that this statement does not depend on the choice of the isomorphisms $Y_{t} \cong Y_{0} \otimes k$ and $X_{t} \cong X_{0} \otimes k$ : note that $\operatorname{Aut}\left(Y_{0}\right)$ acts from the right on $\Psi$, and $\operatorname{Aut}\left(X_{0}\right)$ acts from the left on $\Psi$. As $T$ is connected, and $\sigma(t) \in \Psi$ for every $t$, this shows $\sigma: T \rightarrow G r_{Y_{0}, i} \times T$ to be a constant map; hence there exists $G_{0} \subset Y_{0}$ such that

$$
\operatorname{Ker}\left(\left(\varphi_{T}\right)_{0}\right)=G_{0} \times T \subset \mathcal{Y}\left[p^{N}\right]_{T}=Y_{0} \times T .
$$

As $N \geq n+i$, we see that

$$
\varphi_{T}\left(\mathcal{Y}\left[p^{N}\right]_{T}\right) \supset \mathcal{X}\left[p^{n}\right]_{T}, \quad \text { hence } \mathcal{Y}\left[p^{N}\right]_{T} \supset \mathcal{H}:=\varphi_{T}^{-1}\left(\mathcal{X}\left[p^{n}\right]_{T}\right)=\operatorname{Ker}\left(p^{n} \cdot \varphi_{T}\right) .
$$

We have

$$
\mathcal{X}\left[p^{n}\right]=\mathcal{H} / \mathcal{G} \quad \text { and } \quad G_{0} \subset H_{0} \subset Y_{0} .
$$

We see that $\mathcal{H} \subset \mathcal{Y}\left[p^{N}\right]_{T}=Y_{0} \times T$ defines a section $\Sigma$ of $G r_{Y_{0}, j} \times T$, with $j=h n+i$. Every geometric fiber $\mathcal{H}_{t}$ is the kernel of $p^{n} \cdot \varphi_{t}: Y_{0} \cong \mathcal{Y}_{t} \rightarrow \mathcal{X}_{t} \cong X_{0}$; hence $\mathcal{H}$ defines a section $\Sigma: T \rightarrow G r=G r_{Y_{0}, j} \times T$; this section has the property that for every geometric point $t$ we have $\Sigma(t) \in \Psi\left(Y_{0}, X_{0} ; j\right)$; hence, by the same argument as before,

$\mathcal{H}=H_{0} \times T \subset \mathcal{Y}\left[p^{N}\right]_{T}=Y_{0} \times T, \quad$ with $\mathcal{G} \subset \mathcal{H}$ giving $(\mathcal{G} \subset \mathcal{H})=\left(G_{0} \subset H_{0}\right) \times T$. 
This proves that

$$
\mathcal{X}\left[p^{n}\right]_{T}=\mathcal{H} / \mathcal{G}=\left(H_{0} / G_{0}\right) \times T .
$$

This proves $*(X, n)$ and we have concluded the proof of the theorem.

\section{Central leaves}

2.1. Notation. Let $K \supset \mathbb{F}_{p}$ be a field, and let $X$ be a $p$-divisible group over $K$. Let $S \rightarrow \operatorname{Spec}(K)$ be a noetherian scheme over $K$, and let $\mathcal{Y} \rightarrow S$ be a $p$-divisible group over $S$. We write

$$
\mathcal{C}_{X}(S) \quad:=\left\{s \in S \mid \exists k=\bar{k} \supset \kappa(s), \quad \exists \cong: \mathcal{Y}_{s} \otimes_{\kappa(s)} k \cong X \otimes_{K} k\right\} .
$$

Note that $\mathcal{Y}_{s} \otimes_{\kappa(s)} k \cong X \otimes_{K} k$ as in the definition exists iff there is a field extension $\kappa(s) \subset L$ and an isomorphism $\mathcal{Y}_{s} \otimes_{\kappa(s)} L \cong X \otimes_{K} L$.

Remark. We will write $\mathcal{C}_{X}(\mathcal{Y} \rightarrow S)$ if confusion might be possible. The isomorphism in the definition exists iff such an isomorphisms exists over an algebraic closure of $\kappa(s)$. We have defined $\mathcal{C}_{X}(S) \subset S$ as a subset. Once 2.2 has been proved, working over a perfect field, we consider $\mathcal{C}_{X}(S) \subset S$ as a closed subscheme of $\mathcal{W}_{\mathcal{N}(X)}^{0}(S)$ with the induced reduced scheme structure. Once the theorem has been proved, in such situations the formation $\mathcal{C}_{X}(-)$ commutes with base change.

We remind the reader of the following notation. Let $\beta$ be a Newton polygon (see 7.4), and let $\mathcal{Y} \rightarrow S$ be a $p$-divisible group with the same $d$ and $h=d+c$. We write

$$
\mathcal{W}_{\beta}^{0}(S)=\left\{s \mid \mathcal{N}\left(\mathcal{Y}_{s}\right)=\beta\right\},
$$

and

$$
\mathcal{W}_{\beta}(S)=\left\{s \mid \mathcal{N}\left(\mathcal{Y}_{s}\right) \prec \beta\right\} .
$$

By Grothendieck-Katz (see [10, 2.3.1 and 2.3.2]), we know that $\mathcal{W}_{\beta}(S) \subset S$ is closed and $\mathcal{W}_{\beta}^{0}(S) \subset S$ is locally closed; these sets are given the induced, reduced scheme structure. Note that $\mathcal{C}_{X}(S) \subset \mathcal{W}_{\mathcal{N}(X)}^{0}(S)$.

In this section we will show

2.2. Theorem. Let $K$ be a field, let $X$ be a p-divisible group over $K$. Let $S \rightarrow$ $\operatorname{Spec}(K)$ where $S$ is an excellent scheme over a field $K$. Let $\mathcal{Y} \rightarrow S$ be a p-divisible group. Then

is a closed subset.

$$
\mathcal{C}_{X}(S) \subset \mathcal{W}_{\mathcal{N}(X)}^{0}(S)
$$

2.3. Definition. Let $S$ be a scheme. Let $G_{1} \rightarrow S$ and $G_{2} \rightarrow S$ be finite, locally free group schemes over $S$. Define the "isom-functor" $\mathcal{I}$ by

$$
\forall T \rightarrow S, \quad \mathcal{I}(T)=\operatorname{Isom}\left(G_{1} \times{ }_{S} T, G_{2} \times{ }_{S} T\right) .
$$

2.4. Lemma. This functor is representable by a scheme $I\left(G_{1}, G_{2}\right)=I \rightarrow S$ of finite type over $S$.

Proof. Let $\mathcal{B}_{1}$, respectively $\mathcal{B}_{2}$, be the $\mathcal{O}_{S}$-algebra defining $G_{1} \rightarrow S$, respectively $G_{2} \rightarrow S$. An isomorphism as considered defines an $\mathcal{O}_{T}$-isomorphism $\mathcal{B}_{2} \otimes \mathcal{O}_{T} \rightarrow$ $\mathcal{B}_{1} \otimes \mathcal{O}_{T}$; the functor of such maps is represented by a scheme of finite type over $S$. The condition that such maps moreover define an isomorphism of bialgebras (defining an isomorphism of group schemes) is a closed condition in this scheme. 
2.5. Corollary. With the notation as in 2.2 by 2.4 we see that $\mathcal{C}_{X}(S) \subset S$ is a constructible subset. Hence it contains a dense subset $U \subset \mathcal{C}_{X}(S)$, open in the closure of $\mathcal{C}_{X}(S)$.

Indeed, use 1.7

2.6. Proof of 2.2, Let $S^{\prime}=\operatorname{Zar}\left(\mathcal{C}_{X}(S) \subset \mathcal{W}_{\mathcal{N}(X)(S)}^{0}\right)$ be the Zariski-closure. Let $S^{\prime \prime} \rightarrow S^{\prime}$ be the normalization and $\mathcal{X}^{\prime \prime}=\mathcal{X} \times_{S} S^{\prime \prime}$. Note that $S^{\prime \prime}=\mathcal{W}_{\mathcal{N}(X)}^{0}\left(S^{\prime \prime}\right)$ : the Newton polygon of $\mathcal{X}^{\prime \prime} \rightarrow S^{\prime \prime}$ is constant. By [22] Theorem 2.1] there exists $\varphi: \mathcal{Y} \rightarrow \mathcal{X}^{\prime \prime}$ such that $\mathcal{Y} \rightarrow S^{\prime \prime}$ is completely slope divisible. Let $\operatorname{deg}(\varphi)=p^{i}$. Note that $\mathcal{Y} \rightarrow S^{\prime \prime}$ is geometrically fiberwise constant; see 1.8 Consider $N(h)$ as in 1.7, where $h$ is the height of $\mathcal{X} / S$. Choose $N \in \mathbb{Z}_{>0}$ such that $N \geq N(h)+i$. As $\mathcal{Y} \rightarrow S^{\prime \prime}$ satisfies $*(\mathcal{Y}, N)$ (see 1.4), we can choose a finite surjective $T \rightarrow S^{\prime \prime}$, a field $L$, a morphism $T \rightarrow \operatorname{Spec}(L)$, a point $0 \in T(L)$, and an isomorphism

$$
Y_{0}\left[p^{N}\right] \times T \stackrel{\sim}{\longrightarrow} \mathcal{Y}\left[p^{N}\right] \times_{S^{\prime \prime}} T ;
$$

we fix this isomorphism and write it as an equality.

The morphism $\varphi_{T}: Y_{T} \rightarrow X_{T}$ defines $\mathcal{G}:=\operatorname{Ker}\left(\varphi_{T}\right) \subset \mathcal{Y}\left[p^{N}\right]_{T} \subset Y_{0} \times T$, and this is equivalent to a section

$$
G r_{\mathcal{Y}, i}=G r_{Y_{0}, i} \times T \stackrel{\stackrel{\sigma}{\leftrightarrows}}{\leftrightarrows} T .
$$

Choose $U \subset \mathcal{C}_{X}(S)$ as in 2.5; as $\mathcal{X} \times{ }_{S}\left(U \times_{S} T\right)$ and $\mathcal{Y} \times{ }_{S^{\prime \prime}}\left(U \times_{S} T\right)$ are geometrically fiberwise constant, we conclude that $\sigma$ is a constant map over $U \times{ }_{S} T$; hence it is constant over $T$, and hence there exists $G_{0} \subset Y_{0}$ such that

$$
\operatorname{Ker}\left(\left(\varphi_{T}\right)_{0}\right)=G_{0} \times T \subset \mathcal{Y}\left[p^{N}\right]_{T}=Y_{0} \times T .
$$

As $N \geq N(h)+i$, defining $\mathcal{H}:=\varphi_{T}^{-1}\left(\mathcal{X}\left[p_{T}^{N(h)}\right]\right)$, we see, as in the proof of [1.3, that

for every geometric point $t \in T(k)$ we have $\mathcal{X}_{t}\left[p^{N(h)}\right] \cong X_{0}\left[p^{N(h)}\right] \otimes k$.

By 1.7 this proves that $\mathcal{X}_{t} \cong X_{0} \otimes k$ for every geometric point $t \in T(k)$. Hence $\mathcal{X}_{T} \rightarrow T$ is geometrically fiberwise constant; this proves that $\mathcal{C}_{X}(S)=S^{\prime}$, which finishes the proof of Theorem 2.2 .

2.7. Proposition/Notation $\mathrm{cu}(-)$. (i) Let $\varphi_{0}: Y_{0} \rightarrow X_{0}$ be an isogeny of $p$ divisible groups over a field $k$; let $R$ be a complete local noetherian domain with residue class field $k$ and let $Y \rightarrow T:=\operatorname{Spec}(R)$ be a geometrically fiberwise constantp-divisible group. Consider $C:=\mathcal{C}_{X_{0}}\left(\operatorname{Def}\left(X_{0}\right)\right)$ and the restriction $X \rightarrow C$ of the universal family. There exists a correspondence $T \leftarrow \Gamma \rightarrow C=\mathcal{C}_{X}(\operatorname{Def}(X))$ and an isogeny $\varphi: Y_{\Gamma} \rightarrow X_{\Gamma}$ extending $\varphi_{0}$ with $T \leftarrow \Gamma$ a finite, surjective morphism.

(ii) For every Newton polygon $\beta$ there exists an integer $\mathrm{cu}(\beta) \in \mathbb{Z}_{\geq 0}(\mathrm{c}=$ central leaf, $\mathrm{u}=$ unpolarized) such that for every $p$-divisible group $X$ with $\mathcal{N}(X)=\beta$ the closed locus $\mathcal{C}_{X}(\operatorname{Def}(X))$ is pure of dimension $\operatorname{cu}(\beta)$.

The first part follows from [1.3; for more details see the proof of 3.14 The second part is an immediate consequence of the first.

2.8. Remark. In [1] we prove the following formula. Let $\beta$ be a Newton polygon of dimension $d$ and height $h$, given by the slopes $0 \leq \lambda_{1} \leq \cdots \leq \lambda_{h} \leq 1$. Let $\beta^{*}$ be the (upper convex) polygon given by $\lambda_{h}, \cdots, \lambda_{1}$. We have $\operatorname{cu}(\beta)=$ $\sum_{0<j<h}\left(\beta^{*}(j)-\beta(j)\right)$. 


\section{Central leaves: The polarized Case}

3.1. Let $A \rightarrow S$ be an abelian scheme. Let $\lambda: A \rightarrow A^{t}$ be a polarization on $A \rightarrow S$; this isogeny is symmetric, i.e., $\lambda=\lambda^{t}$ under the identification $A \stackrel{\sim}{\longrightarrow} A^{t t}$.

Let $Y \rightarrow S$ be a $p$-divisible group. Let $Y^{t} \rightarrow S$ be its Serre-dual. We say that an isogeny $\lambda: Y \rightarrow Y^{t}$ is a quasi-polarization on $Y / S$ if

$$
\left(\lambda^{t}: Y^{t t} \rightarrow Y^{t}\right)=-\left(Y^{t t}=Y \stackrel{\lambda}{\longrightarrow} Y^{t}\right) .
$$

Note that a polarization (a symmetric isogeny) on an abelian scheme $A$ induces a quasi-polarization (an anti-symmetric isogeny) on its $p$-divisible group $X=A\left[p^{\infty}\right]$; see [15] Proposition 1.12]; a polarization on $A$ and the induced quasi-polarization on $X=A\left[p^{\infty}\right]$ will be denoted by the same symbol, unless there might be danger of confusion.

3.2. Notation. Let $K \supset \mathbb{F}_{p}$ be a field, and let $(X, \lambda)$ be a quasi-polarized $p$-divisible group over $K$. Let $S \rightarrow \operatorname{Spec}(K)$ be a scheme over $K$, and let $(\mathcal{Y}, \mu) \rightarrow S$ be a quasi-polarized $p$-divisible group over $S$. We write

$$
\mathcal{C}_{(X, \lambda)}(S) \quad:=\left\{s \in S \mid \exists k=\bar{k} \supset \kappa(s), \quad \exists \cong:\left(\mathcal{Y}_{s}, \mu_{s}\right) \otimes_{\kappa(s)} k \cong(X, \lambda) \otimes_{K} k\right\} .
$$

In case $K$ is perfect, we consider $\mathcal{C}_{(X, \lambda)}(S)$ as a scheme with the induced reduced scheme structure.

3.3. Theorem. Let $K$ be a field, and let $(X, \lambda)$ be a quasi-polarized $p$-divisible group over $K$. Let $S \rightarrow \operatorname{Spec}(K)$ where $S$ is an excellent scheme over $K$. Let $(\mathcal{Y}, \mu) \rightarrow S$ be a quasi-polarized p-divisible group over $S$. Then

$$
\mathcal{C}_{(X, \lambda)}(S) \subset \mathcal{W}_{\mathcal{N}(X)}^{0}(S)
$$

is a closed subset, which is a union of connected components of $\mathcal{C}_{X}(S)$.

By 2.2 we know that $\mathcal{C}_{X}(S) \subset \mathcal{W}_{\mathcal{N}(X)}^{0}(S)$ is closed. Take the union of those irreducible components $C \subset \mathcal{C}_{X}(S)$ for which for the generic point $\eta \in C$ an isomorphism $\left(\mathcal{Y}_{\eta}, \mu_{\eta}\right) \otimes_{\kappa(\eta)} k \cong(X, \lambda) \otimes_{K} k$ exists; using Theorem 1.3 and Corollary 1.7] we see that this union equals $\mathcal{C}_{(X, \lambda)}(S)$.

3.4. Central leaves. For any point $[(A, \lambda)]=x \in \mathcal{A}_{g, d} \otimes \mathbb{F}_{p}$ we choose a field $K$ over which $\left(X:=A\left[p^{\infty}\right], \lambda\right)$ is defined, and we consider

$$
C(x):=\mathcal{C}_{(X, \lambda)}\left(\mathcal{A}_{g, d} \otimes K\right) .
$$

Suppose $K$ is perfect; a geometrically irreducible component of $C(x)$ with the induced reduced scheme structure will be called a central leaf. We will see that for $x \in \mathcal{A}_{g, d, n} \otimes k$ with $n \geq 3$ prime to $p$ there is exactly one irreducible component of $C(x)$ containing $x$; this component will be denoted by $C_{x} \subset \mathcal{A}_{g, d, n} \otimes k$. See 6.5

3.5. Notation. Over $\mathbb{F}_{p^{2}}$ we consider two series of quasi-polarized superspecial $p$-divisible groups (see [11, 6.1]:

$\mathbf{I}_{r}:\left(S=G_{1,1}, \tau_{r}\right)$, where on $M=\mathbb{D}(S)=W x \oplus W \cdot \mathcal{F} x$, with $\mathcal{F} x=\mathcal{V} x$ the pairing $\tau_{r}$ is given by

$$
\epsilon \in W-p W, \epsilon^{\sigma}=-\epsilon, \quad\langle x, \mathcal{F} x\rangle=p^{r} \cdot \epsilon, \quad \operatorname{deg}\left(\tau_{r}\right)=p^{2 r}, \quad r \in \mathbb{Z}_{\geq 0} ;
$$

$\mathbf{I I}_{r}:\left(T=\left(\left(G_{1,1}\right)^{2}, \nu_{r}\right)\right)$, where on $M=\mathbb{D}(T)=W x \oplus W \cdot \mathcal{F} x \oplus W y \oplus W \cdot \mathcal{F} y$ the pairing is given by $\langle x, y\rangle=p^{r},\langle\mathcal{F} x, \mathcal{F} y\rangle=p^{r+1}$ (and all other pairs of base vectors pair to zero); here $\operatorname{deg}\left(\nu_{r}\right)=p^{4 r+2}$, and $r \in \mathbb{Z}_{\geq 0}$. 
In [11, 6.1] it is proved that over an algebraically closed field a superspecial quasipolarized $p$-divisible group is isomorphic to a direct sum of summands each of type I or II.

3.6. Notation. Choose coprime, non-negative integers $m, n \in \mathbb{Z}_{\geq 0}$, with $m>n$. For any $r \in \mathbb{Z}_{\geq 0}$ we consider over $\mathbb{F}_{p}$ a quasi-polarized $p$-divisible group $(U, \zeta)$, sometimes written as $U=U(m, n), \quad \zeta=\zeta_{r}(m, n)$ by $U(m, n)=H_{m, n} \oplus H_{n, m}$, and

$$
\zeta=\left(\begin{array}{cc}
0 & -\bar{\pi}^{r} \\
\pi^{r} & 0
\end{array}\right): \quad H_{m, n} \oplus H_{n, m} \quad \longrightarrow \quad\left(H_{m, n} \oplus H_{n, m}\right)^{t},
$$

where $\pi \in \operatorname{End}\left(H_{m, n} \otimes \mathbb{F}_{p}\right)$ is a uniformizer in $\operatorname{End}\left(H_{m, n} \otimes \overline{\mathbb{F}_{p}}\right)$ (see [7. 5.3]) and $\pi=\pi_{m, n}: H_{m, n} \rightarrow H_{m, n}=\left(H_{n, m}\right)^{t}$, and $\bar{\pi}=\pi_{n, m} \in \operatorname{End}\left(H_{n, m} \otimes \mathbb{F}_{p}\right)$ is a uniformizer. Note that $\pi^{t}=\bar{\pi}$ under the identification $\left(H_{m, n}\right)^{t}=H_{n, m}$.

We assume $\operatorname{gcd}\left(m_{i}, n_{i}\right)=1$ and $m_{i}>n_{i}$ and $n_{i} /\left(m_{i}+n_{i}\right)<n_{j} /\left(m_{j}+n_{j}\right)$ for $j>i$.

3.7. Proposition (elementary divisor). In this proposition we work over an algebraically closed field $k$. Suppose $\xi$ is a symmetric Newton polygon, and let $(H, \zeta)$ be a quasi-polarized p-divisible group with

$$
H \cong H(\xi)=\left(\oplus_{i}\left(H_{m_{i}, n_{i}}\right)^{r_{i}}\right) \bigoplus\left(G_{1,1}\right)^{s} \bigoplus\left(\oplus_{i} \quad\left(H_{n_{i}, m_{i}}\right)^{r_{i}}\right)
$$

i.e., $H$ is minimal; see 7.5 . Then there exists an isomorphism

$$
(H, \zeta) \cong \oplus_{i} \oplus_{1 \leq j \leq r_{i}}\left(U_{m_{i}, n_{i}}, \zeta_{d_{i, j}}\left(m_{i}, n_{i}\right)\right) \bigoplus\left(\oplus_{b}\left(Q_{b}, \beta_{b}\right)\right),
$$

where the non-negative integers $d_{i, 1}|\cdots| d_{i, r_{i}}$ are the elementary divisors of the quasi-polarization on this isoclinic part, and every $\left(Q_{b}, \beta_{b}\right)$ is of type $\mathbf{I}$ or of type II as in 3.5.

Proof. It suffices to show this for the supersingular part, and for the parts $\left(H_{m_{i}, n_{i}} \oplus\right.$ $\left.H_{n_{i}, m_{i}}\right)^{r_{i}}$ and $\left(G_{1,1}\right)^{s}$ separately: $\zeta$ is in block form. For the supersingular part this is proven in [11, 6.1, proposition]. A quasi-polarization $\zeta$ on $\left(H_{m_{i}, n_{i}} \oplus H_{n_{i}, m_{i}}\right)^{r_{i}}$ is in block form

$$
\zeta=\left(\begin{array}{cc}
0 & \gamma \\
\beta & 0
\end{array}\right), \quad \beta: H_{m_{i}, n_{i}}^{r_{i}} \rightarrow\left(\left(H_{n_{i}, m_{i}}\right)^{t}\right)^{r_{i}}, \quad \gamma:\left(H_{n_{i}, m_{i}}\right)^{r_{i}} \rightarrow\left(H_{m_{i}, n_{i}}^{t}\right)^{r_{i}}
$$

by $\zeta^{t}=-\zeta$, on $p$-divisible groups, we conclude that this is a quasi-polarization iff $\beta^{t}=-\gamma$. We use that $E=\operatorname{End}\left(H_{m, n} \otimes k\right)$ is the maximal order in $\operatorname{End}^{0}\left(H_{m, n} \otimes k\right)$, and the same for $H_{n, m}$ : by choosing an appropriate basis for $\left(H_{n_{i}, m_{i}}\right)^{r_{i}}$ and for $\left(H_{m_{i}, n_{i}}^{t}\right)^{r_{i}}$ the morphism $\beta \in \mathrm{M}\left(r_{i}, E\right)$ can be diagonalized with only powers of $\pi$ on the diagonal; see [24, Th. 17.7]. This finishes the proof of the proposition.

3.8. Corollary. Over an algebraically closed field there is, up to isomorphism, precisely one principal quasi-polarization on $H(\xi)$.

3.9. Corollary. Let $X$ be a p-divisible group over $k$. The number of principal quasi-polarizations on $X$ up to isomorphism is finite.

Proof. Choose an isogeny $\psi: H=H(\mathcal{N}(X)) \rightarrow X$; write $\operatorname{deg}(\psi)=p^{i}$. Any principal polarization $\lambda$ on $X$ pulls back to a polarization $\psi^{*}(\lambda)$ of degree $p^{2 i}$. The number of polarizations of a given degree on $H$ is finite by [3.7. The isogeny $\psi$ gives an identification $\operatorname{End}^{0}(X) \rightarrow \operatorname{End}^{0}(H)$ by $\gamma \mapsto \psi^{-1} \gamma \psi$; hence we obtain $\operatorname{End}(X) \hookrightarrow \operatorname{End}(H)$; this gives a finite index subgroup $\operatorname{Aut}(X) \rightarrow \operatorname{Aut}(H)$; hence the number of $\lambda$ which give $\zeta=\psi^{*}(\lambda)$ is finite. 
3.10. The central stream. For a symmetric Newton polygon $\xi$ we have defined the minimal $p$-divisible group $H=H(\xi)$. We see that $H$ admits a principal quasipolarization $\zeta$ defined over $\mathbb{F}_{p}$, and we have seen (see 3.8) that it is unique up to isomorphism over an algebraically closed field. We write

$$
\mathcal{Z}_{\xi}:=\mathcal{C}_{H(\xi)}(\mathcal{A})=\mathcal{C}_{(H(\xi), \zeta)}(\mathcal{A}) ;
$$

this will be called the central stream in $\mathcal{A}=\mathcal{A}_{g, 1} \otimes \mathbb{F}_{p}$ defined by $\xi$. There does exist $[(X, \zeta)]=x \in \mathcal{A}$ with $(X, \zeta)\left[p^{\infty}\right] \otimes k \cong(H(\xi), \zeta)$, such that $\zeta$ is a principal polarization on $A$; in this case we have $\mathcal{Z}_{\xi}=C(x)$.

3.11. Remark. There exist $p$-divisible groups over $k$ for which the number of principal quasi-polarizations is bigger than one.

Example. If $X \sim\left(G_{1,1}\right)^{2}$ and $a(X)=1$, the number of principal quasi-polarizations on $X$ over $k$ is bigger than one.

3.12. Proposition. (i) Type $\mathbf{I}_{r}$. There exists an isogeny $F:\left(G_{1,1}, \tau_{r+1}\right) \rightarrow$ $\left(G_{1,1}, \tau_{r}\right)$.

(ii) Type $\mathbf{I I}_{r}$. There exist isogenies $\beta:\left(G_{1,1}, \tau_{r}\right)^{2} \rightarrow\left(\left(G_{1,1}\right)^{2}, \nu_{r}\right)$ and $\gamma$ : $\left(\left(G_{1,1}\right)^{2}, \nu_{r+1}\right) \rightarrow\left(G_{1,1}, \tau_{r}\right)^{2}$.

(iii) There exists an isogeny $(\pi, 1):\left(H_{m, n} \oplus H_{n, m}, \zeta_{r+1}\right) \rightarrow\left(H_{m, n} \oplus H_{n, m}, \zeta_{r}\right)$.

(iv) For quasi-polarized p-divisible groups $(X, \lambda)$ and $(Y, \mu)$ with $\mathcal{N}(X)=\xi=$ $\mathcal{N}(Y)$ over $k$ there exist a quasi-polarization $\zeta$ on $Z:=H(\xi)$ and isogenies

$$
(X, \lambda) \stackrel{\varphi}{\longleftarrow}(Z, \zeta) \stackrel{\psi}{\longrightarrow}(Y, \mu), \quad \text { i.e., } \quad \varphi^{*}(\lambda)=\zeta=\psi^{*}(\mu) .
$$

Proof. Statement (i) follows by a direct verification.

(ii) Choosing $\alpha_{p} \subset\left(G_{1,1}\right)^{2}$ with quotient superspecial, we construct $\beta$; direct verification shows the quotient is of type $\left(\mathrm{I}_{r}\right)^{2}$; e.g., one can choose $\beta=(F, 1)$ : $\left(G_{1,1}\right)^{2} \rightarrow\left(G_{1,1}\right)^{2}$. Choose $\gamma: X \rightarrow\left(G_{1,1}\right)^{2}$ of degree $p$ and $X$ superspecial.

(iii) This follows by direct verification.

(iv) It suffices to show this for the isoclinic parts separately. For the supersingular part this follows from 3.5 and (i) (iii). The non-supersingular case follows from (iv) and 3.7

3.13. Theorem. (i) Let $[(A, \lambda, h)]=x \in \mathcal{A}_{g, d, n} \otimes \mathbb{F}_{p}$; here $d \in \mathbb{Z}_{>0}$, i.e., we consider polarizations of degree $d^{2}$, and $n \in \mathbb{Z}_{\geq 3}$ is not divisible by $p$; suppose $K$ is a perfect field, and suppose that $(X, \lambda)=(A, \lambda)\left[p^{\infty}\right]$ is defined over $K$; we view the locally closed set $\mathcal{C}_{(X, \lambda)}\left(\mathcal{A}_{g, d, n} \otimes K\right)$ as a locally closed subscheme, denoted by $C$, with induced, reduced scheme structure over $K$. For $x, y \in C(k)$ there is an isomorphism of formal schemes

$$
C^{/ x} \cong C^{/ y}
$$

This scheme $C$ is smooth over $K$. Any irreducible component of $\mathcal{W}_{\xi}^{0}\left(\mathcal{A}_{g, d, n} \otimes k\right)$ which contains $x$ also contains $C$.

(ii) Notation $c(-)$. For every symmetric Newton polygon $\xi$ there is a number $c(\xi)$ such that for every $d \in \mathbb{Z}_{>0}$, and every $x \in \mathcal{C}_{(X, \lambda)}\left(\mathcal{A}_{g, d} \otimes \mathbb{F}_{p}\right)$ with $\mathcal{N}(A)=\xi$, the scheme $C$ considered in (i) is pure of dimension $c(\xi)$.

Proof. (i) It suffices to prove part (i) in case we work over $K=k$, an algebraically closed field. Let $\mathcal{D}=\operatorname{Def}(X, \lambda)$ be the formal deformation functor. Consider 
$\mathcal{D}=\operatorname{Spf}(R)$. We know that the universal $p$-divisible group over $\mathcal{D}$ can be given over $\operatorname{Spec}(R)$ (see [6] Lemma 2.4.4 on page 23]); we consider

$$
\mathcal{C}_{(X, \lambda)}(\operatorname{Spec}(R))=E \subset \operatorname{Spec}(R) .
$$

Consider $[(A, \lambda)]=x,[(B, \mu)]=y \in C(k)$, with

$$
(A, \lambda)\left[p^{\infty}\right]=(X, \lambda)=(B, \mu)\left[p^{\infty}\right] .
$$

By a theorem by Serre and Tate (see [9, Th. 1.2.1]), these identifications give isomorphisms

$$
\operatorname{Def}(A, \lambda) \stackrel{\sim}{\sim} \operatorname{Def}(X, \lambda) \stackrel{\sim}{\longrightarrow} \operatorname{Def}(B, \mu) .
$$

These induce identifications

$$
C^{/ x}=E^{/ 0}=C^{/ y}
$$

where $C^{/ x}$ denotes the formal completion of $C$ at the closed point $x \in C$. Hence the reduced scheme $C$ over $k$ is generically regular; hence it is regular.

For $y \in C(k)$ with the indentifications above, we obtain an identification $\mathcal{W}_{\xi}(\operatorname{Def}(A, \lambda)) \cong \mathcal{W}_{\xi}(\operatorname{Def}(B, \mu))$. This proves that the number of irreducible components of the formal completion $\mathcal{W}_{\xi}\left(\mathcal{A}_{g, d, n} \otimes k\right)^{/ y}$ for all $y \in C$ is constant. This proves the last statement in (i).

(ii) It suffices to prove this statement at points over an algebraically closed field $k$. By the proof in (i) it follows that all irreducible components of $\mathcal{C}_{(X, \lambda)}\left(\mathcal{A}_{g} \otimes k\right)$ have the same dimension. Suppose $A\left[p^{\infty}\right]=X$ and $(A, \lambda)=x \in \mathcal{C}_{(X, \lambda)}\left(\mathcal{A}_{g} \otimes k\right)$, and let $(Y, \mu)$ be a quasi-polarized $p$-divisible group with $\mathcal{N}(X)=\xi=\mathcal{N}(Y)$. We are going to show that the dimensions of $\mathcal{C}_{(X, \lambda)}\left(\mathcal{A}_{g} \otimes k\right)$, respectively $\mathcal{C}_{(Y, \mu)}\left(\mathcal{A}_{g} \otimes k\right)$, are the same. (Once this is proven, we see that this dimension only depends on $\mathcal{N}(X)=\xi$, and that number will be called $c(\xi)$.)

By 3.12 we construct isogenies of quasi-polarized $p$-divisible groups

$$
(X, \lambda) \stackrel{\varphi}{\longleftarrow}(Z, \zeta) \stackrel{\psi}{\longrightarrow}(Y, \mu)
$$

we conclude the existence of isogenies of polarized abelian varieties $(A, \lambda) \stackrel{\varphi}{\longleftarrow}$ $(M, \zeta) \stackrel{\psi}{\longrightarrow}(B, \mu)$, with $(M, \zeta)\left[p^{\infty}\right]=(Z, \zeta)$ and $(B, \mu)\left[p^{\infty}\right]=(Y, \mu)$. The claim (ii) follows from the following lemma applied to both $\varphi$ and $\psi$; the lemma says "an isogeny between polarized abelian varieties extends to an isogeny correspondence between the two leaves".

3.14. Lemma. Let $(A, \lambda) \stackrel{\varphi}{\longleftarrow}(M, \zeta)$, an isogeny, and

$$
(A, \lambda)\left[p^{\infty}\right]=(X, \lambda) \stackrel{\varphi}{\longleftarrow}(Z, \zeta)=(M, \zeta)\left[p^{\infty}\right]
$$

be defined over an algebraically closed field $k$; let $\operatorname{deg}(\varphi)=p^{i}$. Write $x=[(A, \lambda)] \in$ $\mathcal{A}_{g, *, n} \otimes k$ (in the notation we omit the level structure with $n \geq 3$ prime to $p$ ), and let $C_{x}$ be the irreducible component of $\mathcal{C}_{(X, \lambda)}(\mathcal{A})$ containing $x$; write $z=[(M, \zeta)]$ and let $C_{z}$ be the irreducible component of $\mathcal{C}_{(Z, \zeta)}(\mathcal{A})$ containing $z$. There exist an isogeny correspondence consisting of a scheme $T$ and finite surjective morphisms $C_{x} \nleftarrow T \rightarrow C_{z}$. Hence $\operatorname{dim}\left(C_{x}\right)=\operatorname{dim}\left(C_{z}\right)$.

Proof. Consider the universal family restricted to this locally closed subscheme $C_{z}$; we obtain $(\mathcal{M}, \zeta) \rightarrow C_{z}$. Consider the universal family restricted to $C_{x}$; we obtain $(\mathcal{P}, \zeta) \rightarrow C_{x}$. Let $h$ be the height of $Z$; choose an integer $N \geq N(h)+i$, where $N(h)$ is as in 1.7 By 1.3 we construct a finite, surjective morphism $h: T \rightarrow C_{x} \subset C(z)$ 
with an isomorphism $\mathcal{M}\left[p^{N}\right]_{T} \cong Z\left[p^{N}\right] \times T$; assume $T$ is irreducible. Let $\operatorname{Ker}(\varphi$ : $\left.\mathcal{M}_{z}=Z \rightarrow X\right)=G$. Note that $G$ is isotropic for the form given by $\zeta$. We obtain $G \times T \subset Z\left[p^{N}\right] \times T \subset \mathcal{M}_{T}$. This gives $\left(\mathcal{X}^{\prime}, \lambda^{\prime}\right) \rightarrow T$ by

$$
\varphi^{\prime}: \mathcal{M}_{T} \rightarrow \mathcal{M}_{t} /(G \times T)=: \mathcal{X}^{\prime}, \quad\left(\varphi^{\prime}\right)^{*}\left(\lambda^{\prime}\right)=\zeta
$$

(note that $\zeta$ descends via $\varphi^{\prime}$ ). This family over $T$ plus the induced level- $n$-structure from $z$ defines a morphism $f^{\prime}: T \rightarrow \mathcal{A}_{g, *, n} \otimes k$.

Write $\left[p^{N(h)}\right]^{-1}(G)=: G^{\prime} \subset Z\left[p^{N}\right]$. For any $t \in T(k)$ we obtain an isomorphism $G^{\prime} / G \cong \mathcal{X}_{t}^{\prime}\left[p^{N(h)}\right]$. By 1.7 we see that morphism $f^{\prime}$ factors through $f: T \rightarrow C_{x} \subset$ $C(x) \subset \mathcal{A}_{g, *, n} \otimes k$. Hence we have constructed

$$
C_{x} \stackrel{f}{\longleftarrow} \quad T \stackrel{h}{\longrightarrow} C_{z} .
$$

We show that $f$ is quasi-finite. It suffices to show that for any point $u \in C_{x}(k)$ the fiber above $u$ in $f: T \rightarrow C_{x}$ is finite. Suppose $S \subset T$ is an irreducible, reduced subscheme with $f(S)=u$; we show that $S$ is a point. For any $y \in C_{x}$ choose an isomorphism $\left(\mathcal{P}_{x}, \lambda\right)\left[p^{\infty}\right] \cong(X, \lambda)$, and for any $t \in C_{z}$ choose an isomorphism $\left(\mathcal{M}_{t}, \lambda\right)\left[p^{\infty}\right] \cong(Z, \lambda)$. Choose $q=p^{m}$ with $m \geq i$, and construct $\Lambda=q^{*}(\lambda)$ and isogenies

$$
(\mathcal{P}, \lambda)_{T} \stackrel{\varphi}{\longleftarrow}(\mathcal{M}, \zeta)_{T} \stackrel{\beta}{\longleftarrow}(\mathcal{P}, \Lambda) \text { with } \varphi \cdot \beta=q .
$$

A point $s \in S \subset T$ gives isogenies $\mathcal{P}_{f(s)} \cong X \rightarrow \mathcal{M}_{s} \cong Z \rightarrow \mathcal{X}_{s}^{\prime} \cong X$. By 1.10 the number of possible kernels of $\beta_{s}: \mathcal{P}_{f(S)} \cong X \rightarrow \mathcal{M}_{s} \cong Z$ is finite; these kernels are in $\mathcal{X}_{S}[q]$; by 1.9 and by the fact that $S$ is irreducible, the resulting morphism $S \rightarrow G r$, in the notation of [1.9, is constant. Hence $(\mathcal{M}, \zeta)_{S} \rightarrow S$ is geometrically fiberwise constant. As $S \rightarrow C_{z}$ is finite, and as this morphism is the moduli map defined by $(\mathcal{M}, \zeta)_{S} \rightarrow S$, we conclude that the image of $S \rightarrow C_{z}$ is finite (hence it is just one point); we conclude that $S$ has dimension zero. We have proved that $f: T \rightarrow C_{x}$ is quasi-finite. We see that the existence of an isogeny $\varphi:(M, \zeta) \longrightarrow(A, \lambda)$ proves $\operatorname{dim}\left(C_{z}\right) \leq \operatorname{dim}\left(C_{x}\right)$.

Let $x^{\prime}=[(A, \lambda)]$. Over $C_{x}$ we have a family $(\mathcal{P}, \Lambda)$ this defines a morphism $C_{x} \rightarrow \mathcal{A}_{g, *}$ which factors through $C_{x^{\prime}}$. This map is injective; hence $\operatorname{dim}\left(C_{x}\right) \leq$ $\operatorname{dim}\left(C_{x^{\prime}}\right)$. The existence of $\beta:(A, \Lambda) \rightarrow(M, \zeta)$ proves by the arguments above that $\operatorname{dim}\left(C_{x^{\prime}}\right) \leq \operatorname{dim}\left(C_{z}\right)$. We conclude that $\operatorname{dim}\left(C_{z}\right)=\operatorname{dim}\left(C_{x}\right)$ and that $f: T \rightarrow C_{x}$ is dominant.

We are going to prove that $f: T \rightarrow C_{x}$ is surjective and finite. As the image $f(T)$ is dense in $C_{x}$ and $f$ is quasi-finite, it suffices to prove the valuative criterion for $f$. We use the notation $q, \beta$ introduced above. Let $K \supset R \rightarrow R / m_{R}=k$ be a discrete valuation ring with field of fractions and residue class field. For any $y \in C_{x}$ we choose a commutative diagram

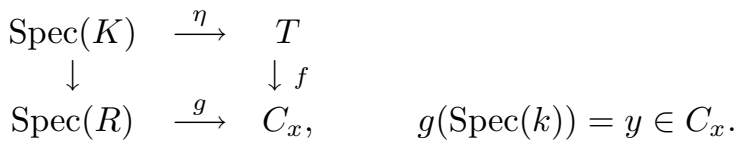

We obtain $\beta_{\operatorname{Spec}(K)}:(\mathcal{P}, \Lambda)_{\operatorname{Spec}(K)} \rightarrow(\mathcal{M}, \zeta)_{\operatorname{Spec}(K)}$. We have $\operatorname{Ker}\left(\beta_{\operatorname{Spec}(K)}\right) \subset$ $(\mathcal{P}, \Lambda)_{\operatorname{Spec}(K)}$; the existence of its flat extension to $(\mathcal{P}, \Lambda)_{\operatorname{Spec}(R)}$ shows that $(\mathcal{P}, \Lambda)_{\operatorname{Spec}(K)} \rightarrow(\mathcal{M}, \zeta)_{\operatorname{Spec}(K)}$ extends to $(\mathcal{P}, \Lambda)_{\operatorname{Spec}(R)} \rightarrow\left(\mathcal{M}^{\prime}, \zeta\right)_{\operatorname{Spec}(R)}$ over $\operatorname{Spec}(R)$. The moduli map defined by $\left(\mathcal{M}^{\prime}, \zeta\right)_{\operatorname{Spec}(R)} \rightarrow \operatorname{Spec}(R)$ by 3.3 lands into $C_{z}$. As $T \rightarrow C_{z}$ is finite, hence proper, we conclude that $\eta$ extends to 
$\operatorname{Spec}(R) \rightarrow T$ leaving the diagram commutative. This proves the valuative criterion for $f: T \rightarrow C_{x}$. Hence $g(\operatorname{Spec}(k))=y \in C_{x}$ is in the image of $f: T \rightarrow C_{x}$; we conclude $f$ is surjective. By the valuative criterion, $f$ is proper; hence $f$ is finite. This ends the proof of the lemma.

In the first part of the theorem we have seen that components of $C(x)$ have the same dimension. Using 3.12 and 3.14, we see that any two central leaves in the same open Newton polygon stratum admit a finite-to-finite isogeny correspondence. Hence, in the notation of the theorem, this proves that $\operatorname{dim}(C(x))=\operatorname{dim}(C(z))=\operatorname{dim}(C(y))$; this concludes the proof of the theorem.

Smoothness as in the theorem of a central leaf was already known in a special case in a different setting; see [4, Coroll. III.4.4 on page 114].

\subsection{We see the curious phenomenon:}

Although the dimension of a component of the Newton polygon strata depends on the degree of the polarization (and there are Newton polygons for which this dimension is not constant inside that stratum; compare 6.4), the dimension of the central leaves in one Newton polygon stratum in $\mathcal{A}_{g} \otimes \mathbb{F}_{p}$ are all of the same dimension.

Moreover, in general a Newton polygon stratum is singular (even after adding level structure, even after considering only one irreducible component of a Newton polygon stratum); however every central leaf (level structure considered) is smooth over the base field.

Isogeny correspondences in general blow up and down (in characteristic $p$ if locallocal kernels are involved); however isogeny correspondences between central leaves are finite-to-finite.

3.16. Remark. Using 3.14 and methods as in [2, VII.3], we can show: Suppose $\varphi$ : $(A, \lambda) \cdots \rightarrow(B, \mu)$ is a quasi-isogeny, then there exists an isogeny correspondence

$$
C_{x} \stackrel{f}{\longleftarrow} \quad T \stackrel{h}{\rightarrow} C_{z}
$$

with $x=[(A, \lambda)]$ and $z=[(B, \mu)]$ which contains the isogeny correspondence $\varphi$ and such that $f$ and $h$ are finite and surjective.

3.17. Remark. In [1] we prove: for a symmetric Newton polygon $\xi$ of height $h=2 g$ we have

$$
c(\xi)=2 \cdot \sum_{0<j \leq g}(\sigma(j)-\xi(j)) .
$$

\section{ISOGENY LEAVES}

Isogeny leaves, as defined below, can be considered as in [23] in the context of "Rapoport-Zink spaces". We have chosen to give an independent exposition.

4.1. Definition. Let $S$ be a scheme over $k$, and let $(\mathcal{D}, \lambda) \rightarrow S$ be a polarized abelian scheme over $S$. A reduced subscheme $I \subset S$ is called an $H_{\alpha}$-subscheme in $S$ if there exists a polarized abelian variety $(M, \zeta)$ over $k$, a scheme $T$ of finite type over $k$, a 
surjective morphism $T \rightarrow I$ and an isogeny $\varphi:(M, \zeta) \times T \rightarrow(\mathcal{D}, \lambda) \times{ }_{I} T$ such that every geometric fiber of $\varphi$ is a successive extension of subfactors isomorphic to $\alpha_{p}$; equivalently, every fiber of $\operatorname{Ker}(\varphi)$ is of local-local type. The pair $(T \rightarrow I, \varphi)$ will be called a chart for the $H_{\alpha}$-scheme.

4.2. Theorem. $\left(I(x)\right.$ : the existence of certain maximal $H_{\alpha}$-subschemes $)$. Consider $d \in \mathbb{Z}_{\geq 1}$; we write $\mathcal{L}:=\mathcal{A}_{g, d} \otimes k$. Let $x \in \mathcal{L}(k)$. There exists a reduced, closed subscheme $I(x) \subset \mathcal{L}$ which is $H_{\alpha}$ such that every irreducible component of $I(x)$ contains $x$ and such that for every integral subscheme $V \subset \mathcal{L}$ we have

$$
V \subset \mathcal{L} \text { is an } H_{\alpha} \text {-subscheme, and } x \in V \quad \Longrightarrow \quad V \subset I(x),
$$

i.e., $I(x)$ is the union of all irreducible $H_{\alpha}$-sets in $\mathcal{L}$ containing $x$.

4.3. Notation. Let $[(A, \lambda)]=x \in \mathcal{A}_{g}(k)$; let $I(x) \subset \mathcal{A}_{g, d} \otimes k$ be as in the theorem. An irreducible component of $I(x)$ with the induced, reduced scheme structure will be called an isogeny leaf. I.e., an isogeny leaf is a maximal, integral $H_{\alpha}$-subscheme of $\mathcal{A}_{g} \otimes k$.

4.4. Corollary. Let $S$ be a scheme over $k$, and let $(\mathcal{D}, \lambda) \rightarrow S$ be a polarized abelian scheme; let $x \in S(k)$. There exist a closed subscheme $I(x) \subset S$ which is $H_{\alpha}$ and which contains all irreducible $H_{\alpha}$-subschemes $V$ such that $x \in V \subset S$.

4.5. Isogeny correspondences. Consider the scheme of all isogeny correspondences

$$
\mathcal{I} \longrightarrow\left(\mathcal{A}_{g, *} \otimes \mathbb{F}_{p}\right) \times\left(\mathcal{A}_{g, *} \otimes \mathbb{F}_{p}\right) .
$$

This scheme is a coarse moduli scheme for all diagrams of isogenies

$$
(A, \lambda) \stackrel{\varphi}{\longleftarrow}(M, \zeta) \stackrel{\psi}{\longrightarrow}(B, \mu) .
$$

See [2, page 251] and the rest of VII.3 and VII.4 in [2] for a discussion. We shall also use this correspondence in case of isogenies between polarized abelian varieties with a level- $n$-structure, with $n$ not divisible by $p$, and considering isogenies of degree prime to $n$. Note:

4.6. Lemma. Let $G \rightarrow S$ be a finite, locally free group scheme of rank $p^{n}$. The set of points $s \in S$ such that $G_{s}$ is a local group scheme is closed.

This is a question in characteristic $p$. The condition $G_{s} \subset G\left[F^{n}\right]$ inside the flat scheme $G \rightarrow S$ is a closed condition.

We define $\mathcal{H}_{\alpha} \subset \mathcal{I}$ to be the reduced subscheme defined by the condition that we consider only isogenies, in the notation above: $\varphi$ and $\psi$, with local-local kernel. By the previous lemma we see that $\mathcal{H}_{\alpha} \subset \mathcal{I}$ is a closed subscheme.

4.7. Corollary. Let $(T \rightarrow I, \varphi)$ be a chart as in the definition 4.1 there exists a factorization $T \rightarrow T^{\prime} \rightarrow I$ and a morphism of charts $(T, \varphi) \rightarrow\left(T^{\prime}, \varphi^{\prime}\right)$ for $I$ such that $T^{\prime} \rightarrow I$ is a proper morphism (of finite type). One can choose $T^{\prime} \rightarrow I$ to be finite above all generic points of $I$.

If $I^{0} \subset S$ is $H_{\alpha}$ as in the definition, then its closure $I \subset S$ is also $H_{\alpha}$.

Every component of $\mathcal{H}_{\alpha}$ is of finite type and proper over both factors.

4.8. Remark. In general, a connected union of $H_{\alpha}$-subschemes need not be a closed subscheme. 
Example. Let $\xi=(2,1)+(1,2)$, and consider the central stream $\mathcal{Z}_{\xi}$. Let $x \in \mathcal{Z}_{\xi}$. Consider all $x^{\prime}$ in $\mathcal{A}=\mathcal{A}_{3,1} \otimes \mathbb{F}_{p}$ obtained over some field by iterated $\alpha_{p}$-isogenies from $x$. One shows that this gives a connected union of $H_{\alpha}$-subschemes; this union is finite, i.e., it is a closed subset, iff $x \in \mathcal{A}\left(\overline{\mathbb{F}_{p}}\right)$. So, in order to produce a nonclosed, connected union of $H_{\alpha}$-subschemes, start with $x \in \mathcal{Z}_{\xi}$ which is not defined over a finite field.

4.9. Lemma. Let $T$ be a connected, integral $k$-scheme; let $Y$ be a p-divisible group over $k$, and let $\mathcal{Z} \rightarrow T$ be a p-divisible group which is geometrically fiberwise constant. Assume there exists an isogeny $\psi: Y \times T \rightarrow \mathcal{Z}$. Then $\mathcal{Z} \rightarrow T$ is constant.

Proof. The finite flat group scheme $\mathcal{G}=\operatorname{Ker}(\psi) \rightarrow T$, say of rank $p^{i}$, defines a section in $G r_{Y, i} \times T \rightarrow T$; see 1.9 By 1.10 this section lands in every fiber in a finite set of closed points $\Psi \subset G r_{Y, i}$; see 1.10 As $T$ is connected, this section is constant. Hence there exists $G \subset Y$ such that $\mathcal{G}=G \times T$. This shows that $\mathcal{Z} \cong(Y / G) \times T$.

4.10. Proof of 4.2. We choose $n \in \mathbb{Z}_{\geq 3}$ not divisible by $p$. Let $\xi$ be the Newton polygon of $x$.

Claim. If $V \subset \mathcal{W}_{\xi}^{0}\left(\mathcal{A}_{g, d, n} \otimes k\right)$ is closed and $H_{\alpha}$, then $V$ is closed and $H_{\alpha}$ in $\mathcal{A}_{g, d, n} \otimes k$.

Let $T^{\prime} \supset V$ be the Zariski closure of $V$ in $\mathcal{A}_{g, d, n} \otimes k$, let $T^{\prime} \rightarrow T$ be the normalization, and let $V^{\prime} \subset T^{\prime}$ be the inverse image of $V$. We find a polarized abelian variety $(M, \zeta)$ and an isogeny $\varphi^{\prime}:(M, \zeta) \times V^{\prime} \rightarrow(\mathcal{D}, \lambda) \times_{V} V^{\prime}$ such that every geometric fiber of $\operatorname{Ker}\left(\varphi^{\prime}\right)=G^{\prime}$ is of local-local type. By [2, Proposition I.2.7] there exists an extension $\varphi:(M, \zeta) \times T^{\prime} \rightarrow(\mathcal{D}, \lambda) \times_{T} T^{\prime}$; it follows that $\operatorname{Ker}(\varphi)$ is of local-local type. Hence the image of $T$ in $\mathcal{A}_{g, d, n}$ is contained in $\mathcal{W}_{\xi}^{0}\left(\mathcal{A}_{g, d, n}\right) \otimes k$; i.e., $V$ is closed and $H_{\alpha}$ in $\mathcal{A}_{g, d, n} \otimes k$, which proves the claim.

Write $x=(A, \lambda, f)$ and let $\xi=\mathcal{N}(A)$. Let $W_{1}, \cdots, W_{s}$ be the irreducible components of $\mathcal{W}_{\xi}^{0}\left(\mathcal{A}_{g, d, n} \otimes k\right)$ which contain the point $x$.

Maximal $H_{\alpha}$-scheme. For every $W_{j}$ we construct a closed subscheme $I_{j}^{\prime}(x) \subset$ $W_{j} \subset \mathcal{A}_{g, d, n} \otimes k$ which is $H_{\alpha}$, such that every irreducible component of $I_{j}^{\prime}(x)$ contains $x$ and such that every $x \in V \subset W_{j}$ which is irreducible and $H_{\alpha}$ is contained in $I_{j}^{\prime}(x)$.

We choose some $1 \leq j \leq t$ and we write $W^{\prime}=W_{j}$. Let $W \rightarrow W^{\prime}$ be the normalization map. Choose $y \in W(k)$ with $y \mapsto x \in W^{\prime}$. Let $\left(\mathcal{U}^{\prime}, \lambda, f\right) \rightarrow \mathcal{L}$ be the universal family; write $(\mathcal{U}, \lambda, f)=\left(\mathcal{U}^{\prime}, \lambda, f\right) \times_{\mathcal{L}} W$.

We show that there exist a polarized abelian scheme $(\mathcal{M}, \zeta, f)$ with level-n-structure over $W$ and an isogeny $\varphi:(\mathcal{M}, \zeta, f) \rightarrow(\mathcal{U}, \lambda, f)$ with local-local kernel over $W$ such that $\mathcal{Z}:=\mathcal{M}\left[p^{\infty}\right]$ is completely slope divisible. In fact this follows from [22] 2.1] applied to $\mathcal{U}\left[p^{\infty}\right]$ : as $W$ is normal there exists an isogeny $\varphi: \mathcal{Z} \rightarrow \mathcal{X}:=\mathcal{U}\left[p^{\infty}\right]$ where $\mathcal{Z} \rightarrow W$ is a completely slope divisible $p$-divisible group; this isogeny defines $\varphi:(\mathcal{M}, \zeta, f) \rightarrow(\mathcal{U}, \lambda, f)$ as desired. 
Let $\operatorname{deg}(\varphi)=p^{i}=q$ (remark: this degree may depend on the choice of the component $\left.W_{j}\right)$. We write $Z=\mathcal{Z}_{y}$ and $(M, \zeta)=(\mathcal{M}, \zeta)_{y}$; hence $M\left[p^{\infty}\right]=Z$. We write $[(M, \zeta, f)]=: m \in \mathcal{A}_{g, d q, n}(k)$. Note that $(\mathcal{M}, \zeta, f) \rightarrow W$ defines a correspondence

$$
\mathcal{A}_{g, d q, n} \otimes k \quad \leftarrow \quad \mathcal{J} \quad \rightarrow \quad W ; \quad \text { define } \quad I_{j}(x)=\mathcal{J}^{-1}(m) \subset W
$$

here $\mathcal{J}^{-1}(m)$ is the projction to $W$ of the inverse image of $m$ in $\mathcal{J}$. Define $x \in$ $I_{j}^{\prime}(x) \subset M^{\prime}=W_{j}$ to be its image in $W^{\prime}$.

Claim. For any irreducible $V \subset W_{j}=W^{\prime} \subset \mathcal{L}$, which is $H_{\alpha}$, with $x \in V$ we have $V \subset I_{j}^{\prime}(x)$. Indeed, consider a chart $\left(T^{\prime} \rightarrow V, \rho\right)$ of finite type for $V$, with $\rho: D \times T^{\prime} \rightarrow \mathcal{U}_{T^{\prime}}^{\prime}$. Let $T$ be an irreducible component of $T^{\prime} \times_{W^{\prime}} W$ mapping onto $V$ which contains a point $t \in T$ with $t \mapsto y \in W$. We have an isogeny $\rho_{T}: D \times T \rightarrow \mathcal{U}_{T}^{\prime}=\mathcal{U}_{T}$, and we have an isogeny $(\mathcal{M} \rightarrow \mathcal{U}) \times_{W} T=\mathcal{M}_{T} \rightarrow \mathcal{U}_{T}$. By 4.9 we conclude that $\mathcal{M}_{T} \rightarrow T$ is constant; in fact, $\mathcal{M}_{T} \cong M \times T$; the polarizations $\zeta_{T}$ and $\zeta \times T$ coincide at $t$, and hence they are equal; thus we have an isogeny $\varphi_{T}:\left(\mathcal{M}_{T}, \zeta_{T}\right) \rightarrow\left(\mathcal{U}_{T}, \lambda_{T}\right)$. We denote by $\mathcal{G}^{\prime} \rightarrow T$ the kernel of the isogeny $\varphi_{T}: \mathcal{M}_{T} \cong M \times T \rightarrow \mathcal{U}_{T}$; this defines a morphism $f: T \rightarrow Q$ such that $f^{*}(\mathcal{G})=\mathcal{G}^{\prime}$. As this is the kernel of an isogeny of polarized abelian schemes, we conclude that $f$ factors: $f: T \rightarrow P \subset Q$. The commutative diagram

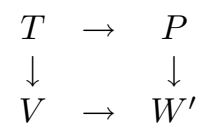

shows that the image is contained in $I_{j}(x)$ :

$$
V=\operatorname{Im}\left(T \rightarrow P \stackrel{\Psi}{\longrightarrow} W^{\prime}\right) \subset I_{j}(x) .
$$

Hence $I_{j}^{\prime}(x)$ thus constructed contains all irreducible $H_{\alpha}$-subschemes contained in $W^{\prime}=W_{j}$ passing through $x$. Hence $I(x):=\cup_{j} I_{j}^{\prime}(x) \subset \mathcal{L} \subset \mathcal{A}_{g} \otimes k$ is $H_{\alpha}$, closed in $\mathcal{A}_{g} \otimes k$, and it contains all irreducible $H_{\alpha}$-subschemes in $\mathcal{A}_{g} \otimes k$ containing $x$. This proves the theorem.

4.11. Proposition. An irreducible, maximal $H_{\alpha}$-scheme $I \subset \mathcal{A}_{g} \otimes k$ is closed in $\mathcal{A}_{g} \otimes k$ and proper over $k$.

Proof. This follows from the definition of maximal $H_{\alpha}$-scheme, and from 1.9 using the valuative criterion of properness.

4.12. Proposition. Let $x \in \mathcal{A}_{g}(k)$ with $x=[(A, \lambda)]$, and $(X, \lambda):=(A, \lambda)\left[p^{\infty}\right]$. Let $[(Y, \mu)]=: y \in C(x)(k)=\mathcal{C}_{(X, \lambda)}\left(\mathcal{A}_{g} \otimes k\right)(k)$. Then there is an isomorphism of formal schemes

$$
I(x)^{/ x} \cong I(y)^{/ y} .
$$

Proof. As $y \in C(x)(k)$, there exists an isomorphism

$$
\varphi:(X, \lambda) \stackrel{\sim}{\longrightarrow}(Y, \mu) .
$$

This gives $\varphi: D_{X}:=\operatorname{Def}(X, \lambda) \stackrel{\sim}{\longrightarrow} D_{Y}:=\operatorname{Def}(Y, \mu)$, and by the Serre-Tate theorem we obtain $\varphi: D_{A}:=\operatorname{Def}(A, \lambda) \stackrel{\sim}{\longrightarrow} D_{B}:=\operatorname{Def}(B, \mu)$.

Claim. Using these isomorphisms, from the facts that $I(x)$ is $H_{\alpha}$ and $I(y)$ is maximally $H_{\alpha}$ at $y$ we can conclude that $\varphi\left(I(x)^{/ x}\right) \subset I(y)^{/ y}$ under the given identifications. 
After considering an appropriate level structure, we conclude we have an isogeny $(M, \zeta) \times I(x) \rightarrow(\mathcal{B}, \lambda)$ expressing that $I(x)$ is $H_{\alpha}$. Let the degree of this isogeny be $p^{i}$. Considering the $p$-divisible groups involved, and restricting to $I(x)^{/ x}$ we obtain an isogeny $(M, \zeta) \times I(x)^{/ x} \rightarrow(\mathcal{D}, \lambda) \times{ }_{D_{A}} I(x)^{/ x}$. Consider $\varphi\left(I(x)^{/ x}\right) \subset I(y)^{/ y} \subset$ $D_{Y}$. Using $\varphi:(X, \lambda) \stackrel{\sim}{\longrightarrow}(Y, \mu)$, we obtain an isogeny $\left(M\left[p^{\infty}\right], \zeta\right) \times \varphi\left(I(x)^{/ x}\right) \rightarrow$ $(\mathcal{Y}, \mu) \times{ }_{D_{Y}} \varphi\left(I(x)^{/ x}\right)$.

Consider the isogeny correspondence (see 4.5) restricted to the factors considered:

$$
\mathcal{I} \subset\left(\mathcal{A}_{g, d, n} \otimes k\right) \times\left(\mathcal{A}_{g, i d, n} \otimes k\right) .
$$

Taking the projection on the second factor to be equal to $(M, \zeta)$ (we omit the level structure in the notation) and only local-local kernels in the isogeny, we obtain a closed set in $\mathcal{I}$ (see 4.6), and using the first projection (which is a proper morphism), we obtain a closed set $T \subset \mathcal{A}_{g, d, n} \otimes k$. By construction it contains the point $y$, it is $H_{\alpha}$, and the completion of $T$ at $y$ contains $\varphi\left(I(x)^{/ x}\right)$. Because $I(y)$ is maximally $H_{\alpha}$ at $y$ we see that every reduced, irreducible component of $T$ containing $y$ is contained in $I(y)$ :

$$
T_{\text {red }} \subset I(y) \text {. }
$$

Hence

$$
\varphi\left(I(x)^{/ x}\right) \subset\left(T_{\text {red }}\right)^{/ y} \subset I(y)^{/ y} .
$$

This proves the claim. Reversing the roles of $x$ and $y$, we show that $\varphi^{-1}\left(I(y)^{/ y}\right) \subset$ $I(x)^{/ x}$. This finishes the proof of the proposition.

4.13. Remark. We did not give a relevant definition of $H_{\alpha}$-schemes for families of $p$-divisible groups; we did not define isogeny leaves in such families. I do not know in which generality an equivalent of 4.2 for $p$-divisible groups holds.

\section{The PROduCt STRUCTURE DEFInED By CENTRAL AND ISOGENY LEAVES}

5.1. Proposition. Let $x \in \mathcal{A}_{g}(k)$. Every irreducible component of $C(x) \cap I(x)$ has dimension equal to zero.

Proof. We will assume considering everything inside $\mathcal{L}:=\mathcal{A}_{g, d, n} \otimes k$, with $n \in$ $\mathbb{Z}_{\geq 3}$ prime to $p$. Let $(\mathcal{U}, \mu, f) \rightarrow \mathcal{L}$ be the universal family. Let $S$ be a reduced, irreducible component of $C(x) \cap I(x)$. There exists a chart of finite type $(T \rightarrow S, \varphi$ : $\left.\left.(M, \zeta) \times T \rightarrow(\mathcal{U}, \mu) \times_{\mathcal{L}} T\right)\right)$. Suppose $T$ is ireducible. As $S \subset C(x)$, we conclude that $\mathcal{U}_{S}$ is geometrically fiberwise constant; hence $(\mathcal{U}, \mu) \times_{\mathcal{L}} T$ is geometrically fiberwise constant. Using 1.9 and 1.10 , we conclude that $(\mathcal{U}, \mu, f) \times_{\mathcal{L}} T$ is constant. Hence the induced moduli morphism $T \rightarrow S \subset \mathcal{L}$ is constant. This proves the proposition.

5.2. Lemma. Let $(Z, \zeta)$ be a quasi-polarized p-divisible group over a field $K$, and let $i \in \mathbb{Z}_{>0}$; write $\mathcal{K}=\operatorname{Ker}(\zeta)$. Consider $G r=G r_{Z, i}$ as in 1.9 with the universal family $\mathcal{G} \rightarrow G r$. The set

$$
P:=\left\{t \in G r \mid \mathcal{G}_{t} \subset \operatorname{Ker}(\zeta) \text {, and it is isotropic for this form }\right\}
$$

is a closed subset of $\mathrm{Gr}$.

Proof. The property $\mathcal{G}_{t} \subset \mathcal{K} \times S \subset Z \times S$ is a closed condition (flat extensions inside a flat $Z \times S \rightarrow S$ ); also the condition that $\mathcal{G}_{S}^{\perp} \subset \mathcal{K} / \mathcal{G}_{S} \subset Z^{t} \times S$ is closed; this proves the claim. 
We show that components of any open Newton polygon stratum (almost) have a product structure given by central laves and isogeny leaves contained in that stratum:

5.3. Theorem ("central and isogeny leaves almost give a product structure on an irreducible component of a Newton polygon stratum"). Let $d \in \mathbb{Z}_{\geq 1}$, let $\xi$ be a symmetric Newton polygon, and let $W^{\prime \prime} \subset \mathcal{A}_{g, d} \otimes k$ be an irreducible component of the open Newton polygon stratum $\mathcal{W}_{\xi}^{0}\left(\mathcal{A}_{g, d} \otimes k\right)$. There exist integral schemes $T$ and $J$ of finite type over $k$ and a finite surjective $k$-morphism

$$
\Phi: T \times J \quad \rightarrow \quad W^{\prime \prime} \subset \mathcal{A}_{g, d} \otimes k
$$

such that

$$
\forall u \in J(k), \quad \Phi(T \times\{u\}) \quad \text { is a central leaf in } W^{\prime \prime},
$$

every central leaf in $W^{\prime \prime}$ can be obtained in this way, and

$$
\forall t \in T(k), \quad \Phi(\{t\} \times J) \quad \text { is an isogeny leaf in } W^{\prime \prime},
$$

and every isogeny leaf in $W^{\prime \prime}$ can be obtained in this way.

Proof. Let $W^{\prime \prime}$ be an irreducible component of $\mathcal{W}_{\xi}\left(\mathcal{A}_{g} \otimes k\right)$; choose $n \geq 3$ prime to $p$, and let

$$
W^{\prime \prime} \leftarrow W^{\prime} \subset \mathcal{W}_{\xi}\left(\mathcal{A}_{g, d, n} \otimes k\right) \subset \mathcal{A}_{g, d, n} \otimes k
$$

be an irreducible (reduced) component of this Newton polygon stratum mapping onto $W^{\prime \prime}$. The finite morphism $\rho: W \rightarrow W^{\prime}$ is the normalization map. Let $(\mathcal{U}, \lambda, f) \rightarrow W$ be the pull back of the universal family. By [22, 2.2], there exists $\varphi:(\mathcal{M}, \zeta, f) \rightarrow(\mathcal{U}, \lambda, f)$ over $W$ such that $\mathcal{Z}:=\mathcal{M}\left[p^{\infty}\right] \rightarrow W$ is completely slope divisible; write $q=p^{i}=\operatorname{deg}(\varphi)$ and $\mathcal{G}=\operatorname{Ker}(\varphi)$. From $(\mathcal{M}, \zeta, f) \rightarrow W$ we obtain the moduli morphism $\pi^{\prime}: W \rightarrow \mathcal{A}_{g, d q, n} \otimes k$.

5.4. Claim/Notation. (i) The image of the moduli morphism $\pi^{\prime}: W \rightarrow \mathcal{A}_{g, d q, n} \otimes k$ is contained in the central leaf $C \subset \mathcal{A}_{g, d q, n} \otimes k$ passing through any point of $\pi^{\prime}(W)$. We obtain

$$
\mathcal{A}_{g, d, n} \otimes k \supset W^{\prime} \stackrel{\rho}{\longleftarrow} \quad W \quad \stackrel{\pi}{\longrightarrow} C \subset \mathcal{A}_{g, d q, n} \otimes k .
$$

(ii) The morphism $\pi: W \rightarrow C$ is proper.

(iii) For any $w \in W(k)$ and any irreducible (reduced) component $J$ of the reduced fiber $\left(W \times_{C}\{\pi(w)\}\right)_{\text {red }} \subset W$ the image $\rho(J) \subset W^{\prime}$ is an isogeny leaf.

(iv) Let $C_{x^{\prime}} \subset W^{\prime}$ be a central leaf and $D$ an irreducible component of $\rho^{-1}\left(C_{x^{\prime}}\right) \subset$ $W$. The morphisms

$$
C_{x^{\prime}} \nleftarrow D \rightarrow C
$$

induced by $\rho$ and by $\pi$ are surjective and finite.

(v) The morphism $\pi: W \rightarrow C$ is surjective.

As $W$ is irreducible, and $\mathcal{Z}:=\mathcal{M}\left[p^{\infty}\right] \rightarrow W$ is completely slope divisible, using 1.8, we conclude that $\pi^{\prime}(W)$ is contained in a central leaf. Note that different central leaves have an empty intersection. Hence there is precisely one central leaf $C$ containing $\pi^{\prime}(W)$; the restriction of $\pi^{\prime}$ we call $\pi: W \rightarrow C$. This proves (i).

Let $R$ be a discrete valuation ring with field of fractions $L$; write $\Gamma=\operatorname{Spec}(R)$ and $\Gamma^{0}=\operatorname{Spec}(L)$; consider a morphism $f: \Gamma \rightarrow C$ such that $f\left(\Gamma^{0}\right) \subset \pi(W)$. We show that also $f(\Gamma) \subset \pi(W)$. Indeed, there exists a finite morphism $g: \Delta \rightarrow \Gamma$ 
(write $\Delta^{0}=g^{-1}\left(\Gamma^{0}\right)$ ) and a morphism $h^{0}: \Delta^{0} \rightarrow W$ with $\pi \cdot h^{0}=\left(\Delta^{0} \rightarrow \Gamma \rightarrow C\right)$. Denote by $\left(\mathcal{M}^{\prime}, \zeta^{\prime}\right) \rightarrow \Delta$ the pull back of the universal family over $\mathcal{A}_{g, d q, n} \otimes k$. The restriction of this to $\Delta^{0}$ is the pull back of $(\mathcal{M}, \zeta) \rightarrow W$. By[1.3] we can suppose that $\Delta$ is choosen in such a way that $\mathcal{M}^{\prime}\left[p^{i}\right]$ is constant over $\Delta$. By 1.10 and 1.7 we see that we obtain $\left(\mathcal{M}^{\prime}, \zeta\right) \rightarrow\left(\mathcal{U}^{\prime}, \lambda\right)$ over $\Delta$, that the moduli map $\Delta^{0} \rightarrow W^{\prime}$ extends, hence $\Delta^{0} \rightarrow W$ extends to $\Delta \rightarrow W$, hence $f(\Gamma) \subset \pi(W)$. Using the valuative criterion (see [5, Th. II.4.7 and Exc. II.4.11]), this shows (ii), that $\pi: W \rightarrow C$ is proper.

Clearly $I:=\rho(J)$ is $H_{\alpha}$ and irreducible. Let $I^{\prime}$ be an isogeny leaf containing $I$; suppose $I \subsetneq I^{\prime}$; then $\operatorname{dim}\left(I^{\prime}\right)>\operatorname{dim}(I)$. In that case there exists $J \subset J^{\prime}$ with $\rho\left(J^{\prime}\right)=I^{\prime}$ and $J^{\prime}$ irreducible; we would obtain $\operatorname{dim}\left(\pi\left(J^{\prime}\right)\right)>0$, and moreover $\pi\left(J^{\prime}\right)$ is $H_{\alpha}$ and $\pi\left(J^{\prime}\right) \subset C$. This is a contradition to 5.1 This proves $I=I^{\prime}$, which proves (iii).

As $W^{\prime} \nleftarrow W$ is finite surjective, the same holds for $C_{x} \nleftarrow D$. Note that $D \rightarrow C$ is quasi-finite: any positive dimensional $D^{\prime} \subset D$ with $\operatorname{dim}\left(\pi\left(D^{\prime}\right)\right)=0$ would give a positive dimensional $\rho\left(D^{\prime}\right) \subset C_{x^{\prime}}$ which is $H_{\alpha}$, a contradiction to 5.1 By (ii) we see that $D \rightarrow C$ is proper and hence finite; $\pi(D) \subset C$ is closed. Moreover $\operatorname{dim}\left(C_{x^{\prime}}\right)=$ $\operatorname{dim}(C)$ by 3.14 Hence $\operatorname{dim}(\pi(D))=\operatorname{dim}(C)$. This proves that $\pi: D \rightarrow C$ is surjective; this proves (iv). As $\pi(D) \subset \pi(W) \subset C$, we have proved (v), and the proof of the claim is finished.

5.5. Construction of $\Phi$. We write $\rho^{\prime \prime}=\left(W \stackrel{\rho}{\longrightarrow} W^{\prime} \rightarrow W^{\prime \prime}\right)$. Choose a point $w \in W(k)$ such that $x^{\prime \prime}:=\rho^{\prime \prime}(w) \in W^{\prime \prime}$ is not contained in any other component of $\mathcal{W}_{\xi}^{0}\left(\mathcal{A}_{g, d} \otimes k\right)$; write $\rho(w)=x^{\prime}$ and $\pi(w)=z=\pi(J)$; choose $J \subset W$ as in (iii) of 5.4 with moreover $w \in J$. We write $M=\mathcal{M}_{w}=\mathcal{M}_{z}^{\prime}$, and $Z=M\left[p^{\infty}\right]$. We see that there exists a finite group scheme, $\mathcal{G} \hookrightarrow M\left[p^{i}\right] \times J$ isotropic under the form given by the polarization $\zeta$ such that $\zeta$ descends to a polarization, say $\mu^{\prime}$, on $(M \times J) / \mathcal{G}$, and such that

$$
\left((M \times J) / \mathcal{G}, \mu^{\prime}\right) \cong(\mathcal{U}, \mu)_{J}
$$

The finite group scheme $\mathcal{G} \hookrightarrow M\left[p^{i}\right] \times J$ defines a morphism $e: J \rightarrow P \subset G r$, in the notation of [5.2; we claim that the morphism e is quasi-finite; indeed, let $V \subset J$ be a closed irreducible subset such that $e(V)$ is a point; this implies that the restriction of $\mathcal{G}$ to $V$ is constant; hence $(\mathcal{U}, \mu)_{V}$ is constant; the fact that the family $(\mathcal{U}, \mu)$ is universal and the fact that $W \rightarrow W^{\prime}$ is finite imply that $V$ is finite; this proves the claim.

Choose $D \subset W$ with $\rho(D)=C_{x^{\prime}}$ and $w \in D$ as in (iv) of 5.4. Using 1.3 we choose a commutative diagram

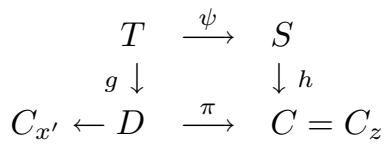

such that $g^{*}(\mathcal{M})\left[p^{i}\right] \cong M\left[p^{i}\right] \times T$, an isomorphism compatible with $h^{*}\left(\mathcal{M}^{\prime}\right)\left[p^{i}\right] \cong$ $M\left[p^{i}\right] \times S$, and such that $T$ is normal and integral. Using the notation $\left(\mathcal{M}^{\prime}, \zeta^{\prime}, f^{\prime}\right)$ for the universal famiily over $\mathcal{A}_{g, d q . n} \otimes k$, we have $g^{*}(\mathcal{M}, \zeta, f)=(h \cdot \psi)^{*}\left(\mathcal{M}^{\prime}, \zeta^{\prime}, f^{\prime}\right)$. 
From

$$
\mathcal{G} \hookrightarrow M\left[p^{i}\right] \times J \quad \text { and } \quad M\left[p^{i}\right] \times T \hookrightarrow \mathcal{M}_{T},
$$

the pull back of the universal family over $G r$ to $J \rightarrow P \subset G r$ with $P$ as in 5.2 respectively the pull back of $\mathcal{M} \rightarrow W$ to $T$ via $T \rightarrow D \subset W$, we obtain

$$
\mathcal{G} \times T \quad \hookrightarrow \quad M\left[p^{i}\right] \times T \times J \quad \hookrightarrow \quad \mathcal{M}_{T} \times J \quad \text { over } \quad T \times J .
$$

By construction this subgroup scheme is contained in the kernel of and isotropic under the form given by $\zeta$; hence we can descend the polarization to the quotient, obtaining

$$
\left(\mathcal{M}_{T} \times J, \zeta_{T} \times J, f\right) \longrightarrow(\mathcal{Q}, \mu, f), \quad \mathcal{Q}:=\left(\mathcal{M}_{T} \times J\right) /(\mathcal{G} \times T)
$$

over $T \times J$. This defines the moduli morphism:

$$
[(\mathcal{Q}, \mu, f)]=\Psi^{\prime}: \quad T \times J \quad \longrightarrow \quad \mathcal{A}_{g, d, n} \otimes k .
$$

Write $\Phi=\left(W^{\prime \prime} \leftarrow W^{\prime}\right) \cdot \Psi^{\prime}$.

5.6. Claim/Notation. (i) We conclude that $\Psi^{\prime}: T \times J \rightarrow W^{\prime}$; this defines a unique $\Psi: T \times J \rightarrow W$ such that $\rho \cdot \Psi=\Psi^{\prime}$; we obtain a commutative diagram:

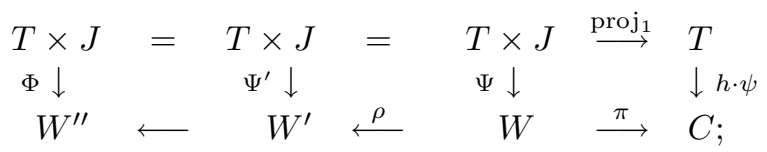

moreover, for every $u \in J(k)$ we have

$$
(T \cong T \times\{u\} \hookrightarrow T \times J \stackrel{\Psi}{\longrightarrow} W)=(T \stackrel{g}{\longrightarrow} D \hookrightarrow W) ;
$$

(ii) the morphism $\Psi: T \times J \rightarrow W$ is finite and surjective; hence $\Phi$ is finite and surjective;

(iii) if $u \in J(k)$, then $\Phi(T \times\{u\})$ is the central leaf passing through $\rho^{\prime \prime}(\Psi(t, u))$ $=\Phi(t, u)$ for any $t \in T(k)$; every central leaf can be obtained in this way;

(iv) if $t \in T(k)$, then $\Phi(\{t\} \times J)$ is an isogeny leaf in $W^{\prime \prime}$; every isogeny leaf can be obtained in this way.

Choose $t_{0} \in T$ with $g\left(t_{0}\right)=w$; choose $u_{0} \in J$ with $e\left(u_{0}\right)=\left[\mathcal{G}_{w}\right] \in P \subset G r$. By construction we see that $\Psi^{\prime}\left(t_{0}, u_{0}\right)=x^{\prime} \subset W^{\prime}$. As the Newton polygon is constant on $\Phi(T \times J)$, and by the choice of $w$, we conclude that $\Psi^{\prime}(T \times J) \subset W^{\prime}$. As $T$ and $J$ are normal, and $\rho: W \rightarrow W^{\prime}$ is the normalization morphism, we obtain $\Psi$ as desired. As $\rho$ is biregular at $w \in W \rightarrow W^{\prime}$, we see that $\Psi\left(t_{0}, u_{0}\right)=w$. Consider the moduli map

$$
\pi^{\prime}=\left[\left(\mathcal{M}_{T} \times J, \zeta_{T} \times J, f\right)\right]: T \times J \rightarrow C ;
$$

for every $t \in T(k)$ this morphism restricted to $\{t\} \times J$ gives one point in $C$; hence it factors over $\operatorname{proj}_{1}: T \times J \rightarrow T$; for every $u \in J(k)$ the restriction of $\left(\mathcal{M}_{T} \times J, \zeta_{T} \times\right.$ $J, f)$ to $T \times\{u\}$ equals $\left(\mathcal{M}_{T}, \zeta_{T}, f\right)$, and hence $\pi^{\prime}$ restricted to $T \times\{u\} \cong T$ equals $h \cdot \psi$. Hence

$$
\pi^{\prime}=h \cdot \psi \cdot \operatorname{proj}_{1} .
$$

We have proved: $(\pi \cdot \Psi)^{*}\left(\mathcal{M}^{\prime}, \zeta^{\prime}\right)=\Psi^{*}(\mathcal{M}, \zeta)=\left(\mathcal{M}_{T} \times J, \zeta_{T} \times J\right)$. This proves that the restriction of $\Psi^{\prime}$ to $T \times\{w\}$ is given by as the moduli map by this family; note that $W^{\prime} \leftarrow W$ is biregular at $w$ by the choice of $w$; hence the last statement in (i) is proved. This shows (i). 
We claim that $\Psi$ is quasi-finite; in fact, let $V^{\prime} \subset T \times J$ be an irreducible, closed subset, such that $\Psi\left(V^{\prime}\right)$ is one point; then it is mapped to one point under $\operatorname{proj}_{1}=$ $t \in T(k)$; hence it is closed in $\{t\} \times J$, i.e., $V^{\prime}=\{t\} \times V$; this means that $e(V)$ is one point, where $e: J \rightarrow P$ is the morphism considered above; we have seen that this implies that $V$ is finite; we conclude that $\Psi$ is quasi-finite.

By dimension arguments we conclude that $\Psi$ is dominant (and we see that $\operatorname{dim}(J)=\operatorname{dim}(W)-\operatorname{dim}(C))$. As $T \rightarrow C$ is finite (hence proper) and $J$ is complete (see 4.11), the composition

$$
(T \times J \rightarrow W \rightarrow C) \quad=(T \times J \rightarrow T \rightarrow C)
$$

is proper. We conclude by [5, II.4.8 (e)] that $T \times J \rightarrow W$ is proper. Hence $\Psi: T \times J \rightarrow W$ is finite and surjective; this shows (ii).

Consider $u \in J(k)$; the restriction of $(\mathcal{U}, \mu, f)$ to $D_{u}:=\Psi(T \times\{u\})$ is geometrically fiberwise constant; hence $\rho\left(D_{u}\right)$ is contained in the central leaf $C^{\prime}:=C_{\rho\left(\Psi\left(t_{0}, u\right)\right)}$ for any $t_{0} \in T(k)$. This image is closed in $C^{\prime}$, and for dimension reasons (see 3.13), $\rho\left(D_{u}\right)$ is dense in $C^{\prime}$; hence equality; hence $\Psi^{\prime}(T \times\{u\})$ is a central leaf.

For any $y^{\prime} \in W^{\prime}$, choose an irreducible component $D^{\prime} \subset \rho^{-1}\left(C_{y^{\prime}}\right)$ and $w^{\prime} \in D^{\prime}$; as $\Psi: T \times J \rightarrow W$ is surjective, we can choose $\left(t_{0}, u_{0}\right) \in T \times J$ with $\Psi\left(t_{0}, u_{0}\right)=w^{\prime}$. We see that $\Psi\left(T \times\left\{u_{0}\right\}\right)=D^{\prime}$; hence the central leaf $C_{y^{\prime}}$ equals $\Psi^{\prime}\left(T \times\left\{u_{0}\right\}\right)$. This proves (iii).

We see that $\pi(\Psi(\{t\} \times J))=z^{\prime}$ is a point. The existence of an irreducible component of $W \times_{C}\left\{z^{\prime}\right\}$ containing $\Psi(\{t\} \times J)$ of strictly bigger dimension would give a contradiction to (ii) of 5.6 , the fact that $T \rightarrow C$ is finite, and 5.1 By (iii) of 5.4 we conclude that $\Phi(\{t\} \times J)$ is an isogeny leaf.

Conversely, let $I^{\prime} \subset I(y) \subset W^{\prime}$ be an isogeny leaf. Choose an irreducible component $J^{\prime} \subset \rho^{-1}\left(I^{\prime}\right)$ and $w^{\prime} \in J^{\prime}(k)$ with $\rho\left(w^{\prime}\right)=y$. We have $\pi\left(J^{\prime}\right)=: z^{\prime} \in C$, and we can choose $\left(t_{0}, u_{0}\right) \in T \times J$ with $\Psi\left(t_{0}, u_{0}\right)=w^{\prime}$, using that fact that $\Psi$ is surjective. As $\pi(\Psi(\{t\} \times J))=z^{\prime}$, and because $\Psi$ is proper and finite, and $\operatorname{dim}\left(J^{\prime}\right)=\operatorname{dim}(J)$, we conclude that $\Psi(\{t\} \times J)=J^{\prime}$. Hence $\Phi(\{t\} \times J)=I^{\prime}$. This shows (iv). This finishes the proof of [5.6, and the proof of Theorem 5.3 is concluded.

5.7. Corollary. Let $W^{\prime \prime}$ be an irreducible component of $\mathcal{W}_{\xi}^{0}\left(\mathcal{A}_{g} \otimes k\right)$.

(i) Let $I \subset W^{\prime \prime}$ be an isogeny leaf and $C \subset W^{\prime \prime}$ a central leaf. Then $C \cap I \neq \emptyset$.

(ii) Every isogeny leaf in $W^{\prime \prime}$ has dimension $\operatorname{dim}\left(W^{\prime \prime}\right)-c(\xi)$.

Indeed, if $I=\Phi(\{t\} \times J)$ and $C=\Phi(T \times\{u\})$, then $\Phi(t, u) \in C \cap I$.

As $\Phi$ is finite and surjective, we obtain $\operatorname{dim}\left(W^{\prime \prime}\right)=\operatorname{dim}(T)+\operatorname{dim}(J)$; for any isogeny leaf $I$ we have $\operatorname{dim}(J)=\operatorname{dim}(\Phi(\{t\} \times J))=\operatorname{dim}(I)$.

5.8. Corollary/Notation $i(-)$. Let $\xi$ be a symmetric Newton polygon (dimension $g$, height $2 g)$. There exists an integer $i(\xi)$ such that for every $x \in \mathcal{A}=\mathcal{A}_{g, 1} \otimes \mathbb{F}_{p}$ the dimension of every geometric irreducible component of $I(x) \subset \mathcal{A}$ equals $i(\xi)$. We have $c(\xi)+i(\xi)=\operatorname{sdim}(\xi)$.

Reminder: The integer $\operatorname{sdim}(\xi)$ was defined in [17, 3.3] and in [19, 1.9]; in [19] Theorem 4.1], we have proved that the dimension of every geometric component of $\mathcal{W}_{\xi}\left(\mathcal{A}_{g, 1} \otimes \mathbb{F}_{p}\right)$ equals this integer: 


$$
\operatorname{dim}\left(\mathcal{W}_{\xi}\left(\mathcal{A}_{g, 1} \otimes \mathbb{F}_{p}\right)\right)=\operatorname{sdim}(\xi) .
$$

5.9. Note that in the last corollary we consider principally polarized abelian varieties. The dimension of a geometric component of an isogeny leaf depends on the component of the Newton polygon stratum containing the leave.

Example. For $\sigma_{3}=\sigma=\xi=3 \cdot(1,1)$ we have $i(\xi)=2$, i.e., isogeny leaves in the supersingular, principally polarized $g=3$ locus all have dimension equal to two; note that any supersingular central leaf is finite; however there do exist supersingular isogeny leaves in $\mathcal{A}_{g, d} \otimes \mathbb{F}_{p}$ of dimension three (see [8, $\left.6.10\left(d=p^{3}\right)\right]$, [11, 12.4 $\left.\left.\left(d=p^{2}\right)\right]\right)$; this produces isogeny leaves in $\mathcal{W}_{\sigma}\left(\mathcal{A}_{3} \otimes \mathbb{F}_{p}\right)$ of dimension bigger than $2=i\left(\sigma_{3}\right)$.

Also for some non-supersingular Newton polygons analogous examples can be given, showing that dimensions of isogeny leaves related to one Newton polygon, contained in different components of $\mathcal{W}_{\xi}^{0}\left(\mathcal{A}_{g} \otimes k\right)$, need not be the same. Compare 6.4 .

5.10. Corollary. Let $I \subset \mathcal{W}_{\xi}\left(\mathcal{A}_{g} \otimes k\right)$ be an isogeny leaf. Then

(i) there is a unique irreducible component $W^{\prime \prime}$ of $\mathcal{W}_{\xi}\left(\mathcal{A}_{g} \otimes k\right)$ containing I;

(ii) there is a dense open subset $U \subset I$ such that for every $y \in U$ we have $I(y)=I$.

Proof. Suppose $I \subset W^{\prime \prime}$, an irreducible component $\mathcal{W}_{\xi}\left(\mathcal{A}_{g} \otimes k\right)$. We consider $w \in W(k)$ as in the proof of Theorem 5.3 in particular such that its image in $W^{\prime \prime} \subset \mathcal{W}_{\xi}\left(\mathcal{A}_{g} \otimes k\right)$ is contained in no other irreducible component of $\mathcal{W}_{\xi}\left(\mathcal{A}_{g} \otimes k\right)$. Write $x^{\prime \prime}=\rho^{\prime \prime}(w)$, and let $C_{x^{\prime \prime}} \subset W^{\prime \prime}$ be the central leaf passing through this point. By the choice of $w$ and by (i) of Theorem 3.13 it follows that $C_{x^{\prime \prime}}$ does not meet any component of $\mathcal{W}_{\xi}\left(\mathcal{A}_{g} \otimes k\right)$ different from $W^{\prime \prime}$. By 5.7 we see that $I \cap C_{x^{\prime \prime}}$ is non-empty; hence $I$ contains at least one point contained in no other component but $W^{\prime \prime}$. This proves (i).

We consider in the proof of Theorem 5.3 in particular and moreover a choice of $w \in W(k)$ such that $W^{\prime}$ is normal at $x^{\prime}=\rho(w)$, i.e., such that $W^{\prime} \leftarrow W$ is birational at $w$. Note that $\left(W^{\prime}\right)^{/ x^{\prime}} \cong\left(W^{\prime}\right)^{/ y}$ for every $y \in C_{x^{\prime}}(k)$; hence $W^{\prime}$ is normal at all points of $C_{x^{\prime}}$. This means that $W^{\prime} \leftarrow W$ is birational at all points above $C_{x^{\prime}}$.

Let $I_{1}^{\prime}, \cdots, I_{s}^{\prime}$ be the irreducible components of the inverse image of $I$ under $W^{\prime \prime} \leftarrow W^{\prime}$. By the previous argument and by 5.7 we conclude that every such component meets the normal locus of $W^{\prime}$. Hence the inverse image of $I$ under $W^{\prime \prime} \leftarrow W$ equals $I_{1} \cup \cdots \cup I_{s}$, the union of the inverse images. For every index $i$ consider $U_{i} \subset I_{i}$ as the set where $I_{i}$ does not meet other irreducible components of the corresponding fiber of $W \rightarrow C$. Let $V^{\prime \prime} \subset I$ be the set of points, images of non-normal points of $W^{\prime}$ intersected with $I$; construct $U$ as the intersection of $I-V^{\prime \prime}$ with all images $\rho^{\prime \prime}\left(U_{i}\right)$. For $y \in U(k)$ an irreducible component of $I(y)$, i.e., an isogeny leaf passing through $y$, is an image of some $\{t\} \times J$, as we know by Theorem [5.3, and hence comes from an irreducible component of the fiber $W \rightarrow C$ above a point $\pi\left(I_{i}^{\prime}\right)$; by the choice of $U$ this means that $I(y)$ is irreducible at $y$, which proves the corollary. 
5.11. Remark. Although an isogeny leaf is contained in a unique component of its Newton polygon stratum, a central leaf may be contained in more than one component of its Newton polygon stratum.

5.12. Remark. Using [1] (see 3.17), we conclude that for symmetric Newton polygons $\xi \varsubsetneqq \xi^{\prime}$ we have $c(\xi)>c\left(\xi^{\prime}\right)$ and $i(\xi) \leq i\left(\xi^{\prime}\right)$.

5.13. Remark. Isogeny leaves, in the context of Rapoport-Zink spaces as in [23, and the product structure as given in 5.3 are studied and described in the case of certain Shimura varieties in [13. It would be worthwhile to describe the product structure in general for all Shimura varieties in mixed characteristic, in particular for the Siegel case studied here.

\section{Some Questions}

6.1. Conjecture (the Hecke Orbit Conjecture). Consider a point $[(A, \lambda)]=x \in$ $\mathcal{A}=\mathcal{A}_{g} \otimes \mathbb{F}_{p}$, and consider its Hecke orbit $\mathcal{H}(x) \subset \mathcal{A}$. We expect this Hecke orbit to be dense in its Newton polygon stratum in the moduli space, i.e., the Zariski closure is expected to be

$$
\overline{\mathcal{H}(x)} \stackrel{?}{=} \quad \mathcal{W}_{\mathcal{N}(A)}(\mathcal{A}) .
$$

Notation: $\mathcal{H}(x)$ is the set of all $y=[(B, \mu)]$ such that there exists a field $L$, an isogeny $\varphi: A_{L} \rightarrow B_{L}$, and an integer $n \in \mathbb{Z}_{>0}$ such that $\varphi^{*}\left(\mu_{L}\right)=n \cdot \lambda_{L}$. We write $y \in \mathcal{H}_{\ell}(x)$ if moreover $\ell$ is a prime number and the degree of $\varphi$ and $n$ are powers of $\ell$.

Note that Hecke- $\ell$-actions "move" in a central leaf: under $\ell$-degree-isogenies, with $\ell \neq p$ the $p$-divisible groups are unchanged. Note that Hecke- $\alpha$-actions (isogenies with local-local kernel) "move" in an isogeny leaf. Moreover we have proved that a non-supersingular Newton polygon in $\mathcal{A}_{g, 1} \otimes \mathbb{F}_{p}$ is geometrically irreducible; see 21]. Therefore Conjecture 6.1 would follow, using 5.3 in case we can prove

6.2. Conjecture. For every prime number $\ell$, different from $p$, the Hecke- $\ell$-orbit $\mathcal{H}_{\ell}(x)$ in $\mathcal{A}=\mathcal{A}_{g, 1} \otimes \mathbb{F}_{p}$ is Zariski-dense in $\mathcal{C}(x)$, a finite union of central leaves.

These conjectured results seem to be true; proofs still have to be written out in full.

6.3. Let $\mathcal{X} \rightarrow S$ be a $p$-divisible group over a scheme over $k$. Define a reduced subscheme $I \subset S$ to be $H_{\alpha}$ if there exists a chart $(T \rightarrow I, \varphi: Y \times T \rightarrow \mathcal{X})$, as in 4.1, where $T \rightarrow I$ is a morphism of finite type. Let $x \in S(k)$.

Question. Does there exist a maximal $I(x) \subset S$ subscheme, as in 4.2, which is $H_{\alpha}$ ?

6.4. Conjecture. Let $\xi$ be a symmetric Newton polygon, with p-rank equal to $f$, i.e., $\xi$ has exactly $f$ slopes equal to zero. We expect that $\mathcal{W}_{\xi}\left(\mathcal{A}_{g}\right)$ has a component of dimension precisely $(g(g-1) / 2)+f$.

Note that it is clear that every such component has at most this dimension; see [14]. 
6.5. Conjecture. Let $\xi$ be a symmetric Newton polygon, of height $2 g$, not equal to the supersingular one: $\xi \varsubsetneqq \sigma$. We expect that $\mathcal{W}_{\xi}\left(\mathcal{A}_{g, 1} \otimes \mathbb{F}_{p}\right)$ is geometrically irreducible. We expect that for every non-supersingular $x \in \mathcal{A}_{g, 1}(k)$ we have $C(x)=$ $C_{x}$; i.e., we expect that $C(x)$ is (geometrically) irreducible.

These conjectured results seem to be true; proofs still have to be written out in full.

6.6. Expectation. Let $\xi$ be a symmetric Newton polygon, of height $2 g$, and let $\eta$ be the generic point of a component of $\mathcal{W}_{\xi}\left(\mathcal{A}_{g} \otimes \mathbb{F}_{p}\right)$; let $X_{\eta}$ be the $p$-divisible group over $k=\overline{\mathbb{F}_{p}(\eta)}$ derived from the geometric fiber over $\eta$. By [25], especially Lemma 9 and Proposition 12, there exists a (canonical, minimal) choice for an isogeny $Z \rightarrow X_{\eta}$ such that $Z$ is completely slope divisible. We expect that $Z_{k} \cong_{k} H(\xi)$; here $H(\xi)$ is the minimal $p$-divisible group with Newton polygon $\xi$; see 7.5

6.7. Let us choose a number $i \in \mathbb{Z}_{>0}$. For any point $[(X, \lambda)]=x \in \mathcal{A}_{g} \otimes \mathbb{F}_{p}$ we can consider $\varphi:=(X, \lambda)\left[p^{i}\right]$, and we can study $S_{\varphi}^{(i)}\left(\mathcal{A}_{g} \otimes \mathbb{F}_{p}\right)$, the set of points $y=[(Y, \mu)]$ such that there exist: an algebraically closed field $k$ over which $y$ is defined and an isomorphism $(X, \lambda)\left[p^{i}\right] \otimes k \cong(Y, \mu)\left[p^{i}\right] \otimes k$; probably this is a locally closed set in $\mathcal{A}_{g} \otimes k$.

Choosing $i=1$, we obtain $S_{\varphi}^{(1)}\left(\mathcal{A}_{g} \otimes \mathbb{F}_{p}\right)=S_{\varphi}$, the EO-strata as defined in [18].

Note that the leaves defined by $S^{(i+1)}$ are contained in leaves defined by $S^{(i)}$ : for $\varphi_{1}, \varphi_{2}, \cdots, \varphi_{i}=\left[(X, \lambda)\left[p^{i}\right]\right], \cdots$ all coming from the same $(X, \lambda)$, we obtain $S_{\varphi_{1}}^{(1)}(-) \supset S_{\varphi_{2}}^{(2)}(-) \supset \cdots$; this descending chain stabilizes after a finite number of steps.

For $i>>1$ we obtain central leaves: given $g$, there exists $N$ such that for every $x$ we have $S_{\varphi_{N}}^{(N)}(-)=\mathcal{C}_{x}(-)$; see1.7.

We studied $S^{(1)}$ in [18], and we consider $S^{(N)}=S^{(\infty)}$ in this paper; one could also study the "intermediate" cases $S^{(i)}$.

6.8. Conjecture. Let $(H(\xi), \zeta)$ be a principally quasi-polarized minimal $p$-divisible group; see 7.5. We expect that the central stream, i.e., the central leaf $\mathcal{Z}_{\xi}=$ $\mathcal{C}_{H(\xi)}\left(\mathcal{A}_{g, 1} \otimes \mathbb{F}_{p}\right)$, is the EO-stratum $S_{H(\xi)[p]}^{(1)}$ associated with $H(\xi)[p]$; conversely, every central leaf equal to its EO-stratum is a central stream. See [20].

6.9. Conjecture. Let $\xi$ be a symmetric Newton polygon, and let $\varphi=\varphi(\xi)$ be the isomorphism class of $H(\xi)[p]$. Let $\psi$ be the isomorphism class of a $\mathrm{BT}_{1}$ such that $S_{\psi}\left(\mathcal{W}_{\xi}^{0}\left(\mathcal{A}_{g, 1} \otimes \mathbb{F}_{p}\right)\right) \neq \emptyset$. We expect that in case $\varphi(\xi)=\varphi \subset \psi$, in the terminology of [18] (14.3)]; this means $S_{\varphi}$ is contained in the Zariski closure of $S_{\psi}$.

6.10. Question. We try to study the closure of central leaves in lower Newton polygon strata. Let $\xi$ be a symmetric Newton polygon, $\mathcal{Z}_{\xi}$ the central stream, and let $C \subset W_{\xi}^{0}$ be a central leaf. It might be that $\mathcal{Z}_{\xi}$ and $C$ have the same boundary inside $\mathcal{A}_{g}$ :

$$
\left(\mathcal{Z}_{\xi}^{c}-\mathcal{Z}_{\xi}\right) \cap \mathcal{A}_{g, 1} \stackrel{?}{=} \quad\left(C^{c}-C\right) \cap \mathcal{A}_{g, 1}
$$

Moreover it could be that for $\xi^{\prime} \prec \xi$ we have $\mathcal{Z}_{\xi^{\prime}} \subset\left(\mathcal{Z}_{\xi}\right)^{c}$; note that we expect in general that a leaf in $W_{\xi^{\prime}}^{0}$ is not in the closure of a leaf in $W_{\xi}^{0}$ (depending on $\xi^{\prime} \prec \xi$ and on which leaf is chosen). 
6.11. We can also study Hecke-correspondences which have only local-étale and étale-local kernels. We denote orbits under such correspondences by $\mathcal{H}_{\text {pna }}(-)$, where $p$ stands for of $p$-power degree and na for non- $\alpha_{p}$. Clearly such correspondences do not change the $p$-divisible group up to isomorphism over $k$. I have no reasonable guess whether an orbit under $\mathcal{H}_{\text {pna }}$ could give a dense subset in a central leaf.

\section{Notations}

7.1. In this paper all base rings and all base schemes will be in characteristic $p$. Usually we will write $K$ for an arbitrary field (in characteristic $p$ ) and $k$ for an algebraically closed field.

7.2. We say that a scheme $S$ satisfies condition (N) if $S$ is integral, and the normalization $S^{\prime} \rightarrow S$ gives a noetherian scheme $S^{\prime}$.

Examples of such schemes: an integral scheme of finite type over a field; an integral Japanese scheme (see [3, IV ${ }^{1}$, Chap. 0, 23.1.1]); an integral excellent scheme (see [3. $\left.\left.\mathrm{IV}^{2} .7 .8\right]\right)$.

7.3. We write $\mathcal{A}_{g} \rightarrow \mathbb{Z}$ for the moduli scheme of polarized abelian schemes of relative dimension $g$. We write $\mathcal{A}$ for the moduli spaces of principally polarized abelian varieties in characteristic $p$ :

$$
\mathcal{A}=\mathcal{A}_{g, 1} \otimes \mathbb{F}_{p}
$$

in some considerations all degrees of polarizations will be allowed. In case we also consider a level structure, we will only consider a level- $n$-structure with $n \in \mathbb{Z}_{>0}$ and prime to $p$. We write $\mathcal{A}_{g, *}=\cup_{d} \mathcal{A}_{g, d}$ and $\mathcal{A}_{g, *, *}=\cup_{d, n} \mathcal{A}_{g, d, n}$.

7.4. Newton polygons. The set of isogeny classes of p-divisible groups over any algebraically closed field is the same as the set of Newton polygons.

This combinatorial, discrete invariant can be described as follows. For given integers $d \in \mathbb{Z}_{\geq 0}$ and $h \in \mathbb{Z}_{\geq d}$, writing $h=d+c$, we will understand under a Newton polygon belonging to $d$ and $h$ :

a polygon starting at $(0,0)$, ending at $(h, c)$,

which is lower convex,

has breakpoints in $\mathbb{Z} \times \mathbb{Z}$,

and which has slopes $0 \leq \lambda \leq 1$.

Note that the set of all $p$-divisible groups over a given algebraically closed field $k$ up to isogeny is in bijective correspondence with the set of all Newton polygons, as was shown in the Dieudonné-Manin theory; see [12. Let us make this correspondence precise. For given non-negative coprime integers $m$ and $n$, we fix a $p$-divisible group $G_{m, n}$ as in $\left[12\right.$; this is defined over $\mathbb{F}_{p}$; this $p$-divisible group has dimension $m$ and its Serre-dual $\left(G_{m, n}\right)^{t}=G_{n, m}$ has dimension $n$. In fact, $G_{1,0}=\mathbb{G}_{m}\left[p^{\infty}\right]$ and $G_{0,1}=\mathbb{Q}_{p} / \mathbb{Z}_{p}$, and for positive $m$ and $n$ the covariant Dieudonné module $\mathbb{D}\left(G_{m, n}\right)$ is generated by one element $e$ satisfying the relation $\mathcal{F}^{m} \cdot e=\mathcal{V}^{n} \cdot e$. To the $p$-divisible group $G_{m, n}$ we associate the Newton polygon $\mathcal{N}\left(G_{m, n}\right)$ consisting 
of $m+n$ slopes equal to $n /(m+n)$;

$$
\begin{gathered}
\operatorname{dim}\left(G_{m, n}\right)=m, \quad \operatorname{dim}\left(\left(G_{m, n}\right)^{t}\right)=n, \\
\operatorname{slope}\left(G_{m, n}\right)=\frac{n}{m+n}, \quad \operatorname{height}\left(G_{m, n}\right)=h=m+n .
\end{gathered}
$$

For any $p$-divisible group $X$ over a field $K$, there exists an isogeny

$$
X \otimes k \sim \bigoplus_{i} G_{m_{i}, n_{i}}
$$

over an algebraically closed field $k$ containing $K$; the slopes $n_{i} /\left(m_{i}+n_{i}\right)$ with multiplicity $m_{i}+n_{i}$ appearing in this sum are arranged in non-decreasing order and they give the Newton polygon of $X$.

Remark. Slopes are defined as, e.g., in [10], 17], [19]. This means: for any slope $\lambda^{\prime}$ appearing in [25] and in [22, the slope defined here is equal to $\lambda=1-\lambda^{\prime}$. In definitions adapted from those papers in this paper we will carry out this adaptation. In this paper a slope filtration, the natural slope filtration and a Newton polygon, is according to non-decreasing slopes: $0 \leq \lambda_{1} \leq \cdots \leq \lambda_{i} \leq \cdots \leq 1$.

Remark. For a scheme $T$ over a scheme $S$ we will write $F_{T / S}=F: T \rightarrow T^{(p)}$ for the relative Frobenius, and for a commutative group scheme we write $V_{G / S}=V$ : $G^{(p)} \rightarrow G$ for the relative Verschiebung. Whenever covariant Dieudonné module theory is considered, we write $\mathcal{V}$ and $\mathcal{F}$ for the Verschiebung and Frobenius maps; note that in the covariant theory $\mathbb{D}(F)=\mathcal{V}$ and $\mathbb{D}(V)=\mathcal{F}$ (in the obvious sense); see [17] Section 1] for an explanation.

Note that we use " $V$-slopes" on $p$-divisible groups and abelian varieties, which is the same as " $\mathcal{F}$-slopes" on Dieudonné modules.

A Newton polygon is called symmetric if any slope $\lambda$ is appearing with the same multiplicity as $1-\lambda$. The Newton polygon of a $p$-divisible group $X$ is symmetric if and only if $X$ and its Serre-dual $X^{t}$ are isogenous. The Newton polygon of (the $p$-divisible group of) an abelian variety $A$ is symmetric: a polarization on an abelian variety by the duality theorem (see $[16,18.1]$ ) gives an isogeny between $A\left[p^{\infty}\right]=: X$ and $X^{t}$. Note that $A^{t}\left[p^{\infty}\right]=\left(A\left[p^{\infty}\right]\right)^{t}$ by the duality theorem; see [16, 19.1].

We write $\gamma \prec \beta$ if these are Newton polygons related to the same numbers $d$ and $c$ and no point of $\gamma$ is below $\beta$ (and we will say " $\gamma$ is lying above $\beta$ ").

Let $\mathcal{X} \rightarrow S$ be a family of $p$-divisible groups, and let $\beta$ be a Newton polygon. We write $\mathcal{W}^{0}(S)$ for the set of points $s \in S$ such that the Newton polygon of $\mathcal{X}_{s}$ equals $\beta$. We write $\mathcal{W}(S)$ for the set of points $s \in S$ such that the Newton polygon of $\mathcal{X}_{s}$ is lying above $\beta$. By Grothendieck-Katz (see [10 Th. 2.3.1]), we know that $\mathcal{W}(S) \subset S$ is closed and $\mathcal{W}^{0}(S) \subset S$ is locally closed.

7.5. Minimal $p$-divisible groups. For a pair $(m, n)$ of coprime non-negative integers we write $H_{m, n}$ for the $p$-divisible group as in [7], 5.3]; $H_{m, n}$ can be defined over $\mathbb{F}_{p}$, it is isogenous with $G_{m, n}$ and over $\overline{\mathbb{F}}_{p}$ its endomorphism ring is the maximal order in its endomorphism algebra; these properties characterize $H_{m, n}$ over an algebraically closed field; also see [20]. Note that $\operatorname{End}\left(H_{m, n} \otimes \mathbb{F}_{p}\right)$ is a commutative algebra, free of rank $h=m+n$ over $\mathbb{Z}_{p}=W\left(\mathbb{F}_{p}\right)$; in this ring we have a uniformizer 
$\pi$ and relations $\pi^{h}=p$ and $\pi^{m}=\mathcal{F}$ and $\pi^{n}=\mathcal{V}$; for any field $K \supset \mathbb{F}_{p^{h}}$ the algebra $\operatorname{End}\left(H_{m, n} \otimes K\right)$ is not commutative if $h=m+n>1$, it is free of rank $h$ over $W\left(\mathbb{F}_{p^{h}}\right)$, it is maximal in its full ring of quotients, and it has $\pi$ as uniformizer.

For a Newton polygon $\beta=\oplus\left(m_{i}, n_{i}\right)$ we write $H(\beta):=\oplus H_{m_{i}, n_{i}}$; this will be called the minimal $p$-divisible group with Newton polygon equal to $\beta$. For any field $K \supset \mathbb{F}_{p}$ we write $H(\beta)$ instead of $H(\beta) \otimes_{\mathbb{F}_{p}} K$, if no confusion can occur.

7.6. Completely slope divisible. We take the definition, due to T. Zink, of a completely slope divisible $p$-divisible group from [25], [22]. This means

Definition. Let $X \rightarrow S$ be a $p$-divisible group. Let $0 \leq t_{1}<t_{2}<\ldots<t_{m} \leq s$ be integers and equalities. A $p$-divisible group $Y$ over a scheme $S$ is said to be completely slope divisible with respect to these integers if $Y$ has a filtration by $p$ divisible subgroups:

$$
0=Y_{0} \subset Y_{1} \subset \ldots \subset Y_{m}=Y
$$

such that the following properties hold:

(1) The quasi-isogenies

$$
\frac{F^{s}}{p^{s-t_{i}}}: Y_{i} \rightarrow Y_{i}^{\left(p^{s}\right)}
$$

are isogenies for $i=1, \ldots, m$.

(2) The induced morphisms

$$
\frac{F^{s}}{p^{s-t_{i}}}: Y_{i} / Y_{i-1} \rightarrow\left(Y_{i} / Y_{i-1}\right)^{\left(p^{s}\right)}
$$

are isomorphisms.

Note that the last condition implies that $Y_{i} / Y_{i-1}$ is isoclinic of exact slope $\lambda_{i}:=$ $t_{i} / s$. See [22, 1.5] for a characterization of a $p$-divisible group over a field being completely slope divisible. Note that we do not suppose that $t_{i}$ and $s$ are relatively prime; it is easy to give an example of a $p$-divisible group over a field which is completely slope divisible with respect to $d \cdot t_{i}$ and $d \cdot s$ where $d \in \mathbb{Z}_{>1}$ and not completely slope divisible with respect to $t_{i}$ and $s$.

7.7. We write $\varphi:(A, \lambda) \rightarrow(B, \mu)$ in case $(A, \lambda)$ and $(B, \mu)$ are polarized abelian schemes over some base $S$, and $\varphi: A \rightarrow B$ is an isogeny such that $\varphi^{*}(\mu)=(\lambda)$. We write $\varphi:(A, \lambda, f) \rightarrow(B, \mu, f)$ in case moreover $f$ is a level structure prime to the characteristic on the base and prime to the degree of $\varphi$. We use analogous notations for $p$-divisible groups.

\section{Some terminology AND AN EXAMPle}

We use: $K$ for a field of characteristic $p$, all base schemes will be in characteristic $p$,

we write $k$ for an algebraically closed field of characteristic $p$; we write $A$ for an abelian variety, $X$ for a $p$-divisible group over a field, and usually $\mathcal{X}$ for a $p$-divisible group over a base of positive dimension; we write $G$ for a finite group scheme,

and usually $\mathcal{G}$ for a group scheme finite and locally free over a base of positive dimension. 
An isoclinic Newton polygon has all slopes equal to each other; the only symmetric isoclinic Newton polygon is the supersingular one: $\sigma=g \cdot(1,1)$.

In this paper we have defined the notion of central leaf; see 2.1, 3.2 and 3.4 We used this terminology "central leaf" in order to contrast with "isogeny leaves". If no isogeny leaves are in consideration in a future publication, one can simply say "leaf" and "foliation" for the notion of central leaves used here.

We think that the central leaf (leaves) connected with a minimal $p$-divisible group (see 7.5) will play an important role; hence we gave this a name, the "central stream", in order to distinguish this from the other central leaves.

For "central leaf", $C(x)$ and $C_{x}$, see [3.4 for "isogeny leaf" and $I(x)$, see 4.3 for "central stream", see 3.10 for "geometrically fiberwise constant", see 1.1; for "constant", see 1.2 for "completely slope divisible", see 7.6. for "minimal", see 7.5] for $\operatorname{dim}(\beta)$, see [19, 1.6]; for $\operatorname{sdim}(\xi)$, see [19, 1.9]; for $\operatorname{cu}(\beta)$, see 2.7; for $c(\xi)$, see 3.13, for $i(\xi)$, see 5.8

8.1. As an illustration we record for $g=4$ the various data considered:

\begin{tabular}{|c|l|c|c|c|c|c|}
\hline $\mathrm{NP}$ & $\xi$ & $f$ & $\operatorname{sdim}(\xi)$ & $c(\xi)$ & $i(\xi)$ & $\mathrm{ES}(H(\xi))$ \\
\hline$\rho$ & $(4,0)+(0,4)$ & 4 & 10 & 10 & 0 & $(1,2,3,4)$ \\
\hline$f=3$ & $(3,0)+(1,1)+(0,3)$ & 3 & 9 & 9 & 0 & $(1,2,3,3)$ \\
\hline$f=2$ & $(2,0)+(2,2)+(0,2)$ & 2 & 8 & 7 & 1 & $(1,2,2,2)$ \\
\hline$\beta$ & $(1,0)+(2,1)+(1,2)+(0,1)$ & 1 & 7 & 6 & 1 & $(1,1,2,2)$ \\
\hline$\gamma$ & $(1,0)+(3,3)+(0,1)$ & 1 & 6 & 4 & 2 & $(1,1,1,1)$ \\
\hline$\delta$ & $(3,1)+(1,3)$ & 0 & 6 & 5 & 1 & $(0,1,2,2)$ \\
\hline$\nu$ & $(2,1)+(1,1)+(1,2)$ & 0 & 5 & 3 & 2 & $(0,1,1,1)$ \\
\hline$\sigma$ & $(4,4)$ & 0 & 4 & 0 & 4 & $(0,0,0,0)$ \\
\hline
\end{tabular}

Here $\rho \succ(f=3) \succ(f=2) \succ \beta \succ \gamma \succ \nu \succ \sigma$ and $\beta \succ \delta \succ \nu$. The notation ES, encoding the isomorphism type of a $\mathrm{BT}_{1}$ group scheme, is as in [18; the number $f$ indicates the $p$-rank.

\section{ACKNOWLEDGMENTS}

I thank Thomas Zink for his ideas; suggestions by him have been incorporated into this paper. Most especially his definition of completely slope divisible $p$ divisible groups, his paper [25] and our cooperations on [22] have been of value to me in preparing this paper. I thank Johan de Jong for discussions while preparing this paper and for careful reading of the manuscript. Some aspects of this paper have already been incorporated by Elena Mantovan into her Harvard PhD thesis (see [13]) after discussions we had in the fall of 2000 and in the spring of 2002. I am grateful to MIT, where part of this paper was written.

\section{REFERENCES}

1. C.-L. Chai and F. Oort, Canonical coordinates on leaves of p-divisible groups. [In preparation]

2. G. Faltings and C.-L. Chai, Degeneration of abelian varieties. Ergebnisse Bd 22, Springer Verlag, 1990. MR 92d:14036

3. A. Grothendieck and J. Dieudonné, Elements de géométrie algébriques. Ch. 0 (suite): IV ${ }^{1}$, Publ. Math. IHES 20 (1964); Ch. IV ${ }^{2}$ : Etude locale des schémas et des morphismes de schémas. Publ. Math. IHES 24 (1965). MR 30:3885. MR 33:7330 
4. M. Harris and R. Taylor, The geometry and cohomology of some simple Shimura varieties. Ann. of Math. Studies 151, Princeton Univ. Press 2001. MR 2002m:11050

5. R. Hartshorne, Algebraic Geometry. Grad. Texts 52; Springer - Verlag 1977. MR 57:3116

6. A. J. de Jong, Crystalline Dieudonné module theory via formal rigid geometry. Publ. Math. IHES 82 (1995), 5-96. MR 97f:14047

7. A. J. de Jong and F. Oort, Purity of the stratification by Newton polygons. Journ. A.M.S. 13 (2000), 209-241. MR 2000m:14050

8. T. Katsura and F. Oort, Supersingular abelian varieties of dimension two or three and class numbers. Algebraic Geometry, Sendai 1985 (Ed., T. Oda). Adv. Stud. in Pure Math. 10 (1987), Kinokuniya Cy Tokyo and North-Holland Cy Amsterdam, 1987; pp. 253-281. MR 90c: 14027

9. N. M. Katz, Serre-Tate local moduli. In: Surfaces algébriques (Sém. de géom. algébr. d'Orsay 1976-78). Lecture Notes Math. 868, Springer - Verlag 1981; Exp. V-bis, pp. 138-202. MR 83k:14039b

10. N. M. Katz, Slope filtration of F-crystals. Journ. Géom. Alg. Rennes, Vol. I, Astérisque 63 (1979), Soc. Math. France, 113-164. MR 81i:14014

11. K.-Z. Li and F. Oort, Moduli of supersingular abelian varieties. Lecture Notes Math. 1680, Springer 1998; 116 pp. MR 99e:14052

12. Yu. I. Manin, The theory of commutative formal groups over fields of finite characteristic. Usp. Math. 18 (1963), 3-90; Russ. Math. Surveys 18 (1963), 1-80. MR 28:1200

13. E. Mantovan, On certain unitary group Shimura varieties. Harvard PhD thesis, April 2002. [To appear]

14. P. Norman and F. Oort, Moduli of abelian varieties. Ann. Math. 112 (1980), 413-439. MR 82h:14026

15. T. Oda, The first de Rham cohomology group and Dieudonné modules. Ann. Scient. Ec. Norm. Sup. 2 (1969), 63-135. MR 39:2775

16. F. Oort, Commutative group schemes. Lecture Notes Math. 15, Springer - Verlag 1966. MR 35:4229

17. F. Oort, Newton polygons and formal groups: conjectures by Manin and Grothendieck. Ann. Math. 152 (2000), 183-206. MR 2002e:14075

18. F. Oort, A stratification of a moduli space of polarized abelian varieties. In: Moduli of abelian varieties. (Eds., C. Faber, G. van der Geer, F. Oort). Progress Math. 195, Birkhäuser Verlag 2001; pp. 345-416. MR 2002b:14055

19. F. Oort, Newton polygon strata in the moduli space of abelian varieties. In: Moduli of abelian varieties. (Eds., C. Faber, G. van der Geer, F. Oort). Progress Math. 195, Birkhäuser Verlag 2001; pp. 417 - 440. MR 2002c:14069

20. F. Oort, Minimal p-divisible groups. [To appear in Ann. Math.]

21. F. Oort, Irreducibility of Newton polygon strata. [In preparation]

22. F. Oort and Th. Zink, Families of p-divisible groups with constant Newton polygon. Documenta Mathematica 7 (2002), 183-201, see

http://www.mathematik.uni-bielefeld.de/documenta/vol-07/09.html MR 2003m:14066

23. M. Rapoport and Th. Zink, Period spaces for p-divisible groups. Ann. of Math. Studies 141, Princeton Univ. Press 1996. MR 97f:14023

24. I. Reiner, Maximal orders. Academic Press, 1975. MR 52:13910

25. Th. Zink, On the slope filtration. Duke Math. J. 109 (2001), 79-95. MR 2003d:14055

Mathematisch Instituut, Postbus 80.010, NL-3508 TA Utrecht, The Netherlands

E-mail address: oort@math.uu.nl 